\title{
A Criterion for Asymptotic Completeness in Local Relativistic QFT
}

\section{Journal Article}

\section{Author(s):}

Dybalski, Wojciech; Gérard, Christian

Publication date:

2014-12

Permanent link:

https://doi.org/10.3929/ethz-b-000090609

Rights / license:

In Copyright - Non-Commercial Use Permitted

Originally published in:

Communications in Mathematical Physics 332(3), https://doi.org/10.1007/s00220-014-2069-y 


\title{
A Criterion for Asymptotic Completeness in Local Relativistic QFT
}

\author{
Wojciech Dybalski ${ }^{1,2}$, Christian Gérard ${ }^{3}$ \\ 1 Institut für Theoretische Physik, ETH Zürich, 8093 Zurich, Switzerland \\ 2 Zentrum Mathematik, Technische Universität München, 85747 Garching, Germany. \\ E-mail: dybalski@ma.tum.de \\ 3 Département de Mathématiques, Université de Paris XI, 91405 Orsay Cedex, France. \\ E-mail: christian.gerard@math.u-psud.fr
}

Received: 4 September 2013 / Accepted: 11 October 2013

Published online: 14 May 2014 - (C) Springer-Verlag Berlin Heidelberg 2014

\begin{abstract}
We formulate a generalized concept of asymptotic completeness and show that it holds in any Haag-Kastler quantum field theory with an upper and lower mass gap. It remains valid in the presence of pairs of oppositely charged particles in the vacuum sector, which invalidate the conventional property of asymptotic completeness. Our result can be restated as a criterion characterizing a class of theories with complete particle interpretation in the conventional sense. This criterion is formulated in terms of certain asymptotic observables (Araki-Haag detectors) whose existence, as strong limits of their approximating sequences, is our main technical result. It is proven with the help of a novel propagation estimate, which is also relevant to scattering theory of quantum mechanical dispersive systems.
\end{abstract}

\section{Introduction}

The physical interpretation of local relativistic quantum field theories (QFT) in terms of particles is a long-standing open problem. The only known class of non-trivial asymptotically complete models are the recently constructed two-dimensional theories with factorizing $S$-matrices [Le08, Ta13]. In the thoroughly studied $P(\phi)_{2}$ models only partial results on asymptotic completeness (AC) of two- and three-particle scattering have been found [SZ76,CD82]. The progress on this fundamental problem is hindered by several conceptual and technical difficulties:

(1) On the conceptual side we face a difficulty which is typical for QFT: the algebra of the observables of a system with infinitely many degrees of freedom may have many non-equivalent representations ('sectors') labelled by some 'charge' [DHR71,DHR74,BF82]. Thus the vacuum sector, whose Hilbert space $\mathcal{H}$ consists of states of zero charge, may contain collections of charged particles whose total charge is zero, for example pairs of oppositely charged excitations. As such configurations do not belong to the subspace $\mathcal{H}^{+}$of Haag-Ruelle scattering states of neutral particles, they undermine the conventional AC relation: 


$$
\mathcal{H}^{+}=\mathcal{H}
$$

inherited from quantum mechanics.

(2) Even if a theory has trivial superselection structure, or all its superselection sectors are properly taken into account, the conventional AC may fail due to the presence of (unphysical) states with too many local degrees of freedom, which do not admit any particle interpretation. This is the case in certain generalized free fields [Gre61, HS65].

(3) On the technical side the main stumbling block is our poor understanding of dynamics of dispersive systems, i.e., systems of particles with non-quadratic dispersion relations. We recall in this context that the classical results on the $n$-body $\mathrm{AC}$ in quantum mechanics [SiSo87, Gr90,De93] do not apply to such theories.

In essence, the first two problems above mean that it is not possible to prove conventional AC from the Haag-Kastler postulates, since there exist counterexamples of physical (1) and unphysical (2) type. It is at best possible to formulate criteria which characterize a class of theories for which (1.1) holds. A search for such conditions, initiated almost half a century ago in [HS65] and continued in [Bu87, Bu94, BS05], has so far been unsuccessful. In the present work we formulate a model-independent criterion for conventional AC in massive Haag-Kastler QFT. Our analysis can be summarized as follows: To tackle difficulty (1), we introduce a 'charged particles free' subspace $\mathcal{H}^{\text {cpf }} \subset \mathcal{H}$, defined in (1.11) below. This subspace is constructed with the help of suitable asymptotic observables (generalizations of the Araki-Haag detectors [AH67]), sensitive only to neutral particles. We formulate a generalized (weaker) concept of AC, suitable for theories with non-trivial superselection structure, which requires that

$$
\mathcal{H}^{+}=\mathcal{H}^{\mathrm{cpf}}
$$

We show that this variant of AC holds in any Haag-Kastler QFT with an upper and lower mass gap, as defined in Sect. 2.1 below. This class includes non-trivial models, as for example $\lambda \phi_{2}^{4}$ and $\lambda \phi_{3}^{4}$ theories at small $\lambda$ [GJS73,GJS74,Bur77]. Incidentally, relation (1.2) shows that also the unphysical states of type (2) are eliminated from the 'charged particles free' subspace. Equality (1.2) can immediately be reformulated as a criterion for conventional AC:

$$
\mathcal{H}=\mathcal{H}^{\mathrm{cpf}} \Leftrightarrow \mathcal{H}^{+}=\mathcal{H} .
$$

Our proof of relations (1.2), (1.3) relies on deep similarities between non-relativistic and relativistic scattering theory brought to light in our recent work [DG12]. They allow us to apply powerful quantum-mechanical techniques, as for example the method of propagation estimates [SiSo87], in the relativistic setting. At this technical level we encounter difficulty (3): The approach of Graf [Gr90], which relies on a phase space propagation estimate, does not apply in the presence of three or more particles with relativistic dispersion relations. We solve this problem with the help of a novel propagation estimate (Proposition 5.3) which is the main technical result of this work. We expect that it will also find applications in scattering theory of non-relativistic dispersive systems [Zi97, Ge91].

The question if criterion (1.3) is useful for proving conventional $\mathrm{AC}$ in concrete interacting models is left open in the present work. Nevertheless, let us provide several remarks on this point which may indicate directions of future research: For theories with trivial superselection structure we expect that our criterion is sharp in the sense 
that it only eliminates unphysical examples of type (2). We recall in this context that general conditions for the absence of Doplicher-Haag-Roberts (DHR) sectors in twodimensional massive theories were given in [Mu98]. These conditions (Haag duality for double cones and split property for wedges) offer a more specific framework for future investigations of the problem of AC in concrete interacting theories. A class of examples which should fit into this setting are the $P(\phi)_{2}$ models in the one-phase region. ${ }^{1}$

Theories with non-trivial superselection structure should be embedded into larger theories, which take all the superselection sectors into account, before criterion (1.3) is checked. Such an embedding can, in principle, be accomplished for any massive Haag-Kastler QFT by a suitable variant of the DHR construction [DHR71, DHR74]. In particular, for massive theories in physical spacetime this procedure is very well understood [BF82] and allows for a construction of Haag-Ruelle scattering states involving both neutral and charged particles. We recall, however, that the resulting larger theory contains charge carrying fields whose commutation and localization properties may significantly differ from the familiar properties of observables: In physical spacetime they may have Fermi statistics and/or string-like localization. In spacetimes of lower dimension braid group statistics [FRS89] or soliton sectors [Fr76,BFG78] may appear. The question of validity of relations (1.2), (1.3) in the presence of these interesting complications is left for future work. Examples of interacting theories with non-trivial superselection structure (soliton sectors) are the $P(\phi)_{2}$ models in the two-phase region. ${ }^{2}$

To outline the construction of the 'charged particles free' subspace $\mathcal{H}^{\text {cpf }}$, appearing in relations (1.2) and (1.3), we need some preparations. The restrictive form of the spectrum condition, which we adopt in this work, is important for this discussion: We assume that the spectrum of the energy-momentum operators, denoted $\mathcal{S} p U$, consists of an isolated simple eigenvalue at zero, corresponding to the vacuum vector $\Omega$, an isolated mass hyperboloid $H_{m}:=\left\{(E, p) \in \mathbb{R}^{1+d}: E=\omega(p)\right\}, \omega(p):=\sqrt{p^{2}+m^{2}}$, carrying neutral single-particle states of mass $m>0$ and a multiparticle spectrum $G_{2 m}$ whose lower boundary is $H_{2 m}$. For precise definitions of other concepts appearing in the discussion below the reader should consult Sect. 2.

Let us fix an energy-momentum vector $\tilde{p}=(E, p) \in H_{m}$, and construct timedependent families of observables $t \mapsto C_{t}$ which are the main building blocks of $\mathcal{H}^{\text {cpf }}$ : We choose an almost-local operator $B$ from the algebra of observables $\mathfrak{A}$ of our theory, s.t. its energy-momentum transfer belongs to a small neighbourhood of $-\tilde{p}$. Denoting by $B(t, x)$ the translation of $B$ by the spacetime vector $(t, x)$ and choosing a suitable function on the phase space $h \in C_{0}^{\infty}\left(T^{*} \mathbb{R}^{d}\right)$ we set

$$
C_{t}:=\int h_{t}^{\mathrm{w}}(x, y) B^{*}(t, x) B(t, y) d x d y
$$

where $h_{t}(x, \xi):=h(x / t, \xi), h_{t}^{\mathrm{w}} \in B\left(L^{2}\left(\mathbb{R}^{d}\right)\right)$ is the Weyl quantization of the symbol $h_{t}$ and $h_{t}^{\mathrm{w}}(x, y)$ is its integral kernel. The function $h$ essentially has the form $h(x, \xi)=$ $h_{0}(x) \chi(x-\nabla \omega(\xi))$, where $h_{0} \in C_{0}^{\infty}\left(\mathbb{R}^{d}\right)$ is supported in a small neighbourhood of the point $\nabla \omega(p)$ and $\chi$ is supported in a small neighbourhood of zero.

Let us now justify that $t \mapsto C_{t}$ can be interpreted for large $t$ as a detector sensitive only to neutral particles whose energies-momenta belong to a small neighbourhood of $\tilde{p}$. By computing the limit $C^{+}$of $t \mapsto C_{t}$, as $t \rightarrow \infty$ on the subspace of Haag-Ruelle

\footnotetext{
1 Split property for wedges is expected but not known yet in these theories. Cf. Section 7 of [Mu98].

2 We refer to the Appendix of [SW] and references therein for a discussion of superselection structure and its relation to the problem of $\mathrm{AC}$ in $P(\phi)_{2}$ models.
} 
scattering states involving both neutral and charged particles, one obtains a counterpart of formula (28) from [AH67]:

$$
C^{+}=(2 \pi)^{d} \sum_{q, q^{\prime}} \int d \xi h\left(\nabla \omega_{q}(\xi), \xi\right)\left\langle\xi, q\left|B^{*} B\right| \xi, q^{\prime}\right\rangle a_{+, q}^{*}(\xi) a_{+, q^{\prime}}(\xi),
$$

where $\omega_{q}(\xi):=\sqrt{\xi^{2}+m_{q}^{2}}, m_{q}$ is the mass of a particle of type $q,|\xi, q\rangle$ its plane-wave configuration with momentum $\xi$ and $a_{+, q}^{*}(\xi)$ the asymptotic creation operator of such a configuration, given by the Haag-Ruelle theory. The sum in (1.5) extends over all pairs $q, q^{\prime}$ s.t. $m_{q}=m_{q^{\prime}}$. In view of the relation $h\left(\nabla \omega_{q}(\xi), \xi\right)=h_{0}\left(\nabla \omega_{q}(\xi)\right) \chi\left(\nabla \omega_{q}(\xi)-\right.$ $\nabla \omega(\xi))$ and of the support properties of $h_{0}$ and $\chi$, the function $\xi \mapsto h\left(\nabla \omega_{q}(\xi), \xi\right)$ is non-zero only for such $\xi$ that $\left(\omega_{q}(\xi), \xi\right)$ is in a small neighbourhood of $\tilde{p}$. (In particular, $m_{q}$ must be close to $m$ ). For such $\xi$ we also have

$$
B|\xi, q\rangle=|\Omega\rangle\langle\Omega|B| \xi, q\rangle,
$$

since the energy-momentum transfer of $B$ is close to $-\tilde{p}$ and $m_{q} \geq m$ for all $q \cdot{ }^{3}$ If the particle of type $q$ is neutral, we can easily find $B$, within the above restrictions, s.t. $\langle\Omega|B| q, \xi\rangle \neq 0$. However, if the particle of type $q$ is charged, we have $\langle\Omega|B| q, \xi\rangle=0$, since observables cannot create charged states from the vacuum. Hence, the sum in (1.5) extends only over neutral particle types and the integral over such $\xi$ that $(\omega(\xi), \xi)$ is in a small neighbourhood of $\tilde{p}$. Thus any non-zero vector from the range of $C^{+}$, on the subspace of Haag-Ruelle scattering states, contains a neutral particle whose energymomentum vector is in a small neighbourhood of $\tilde{p}$ (and possibly some other neutral or charged particles).

We mention as an aside that for a symbol $h(x, \xi)=h_{0}(x)$ we recover from (1.4) a time-dependent family of observables of the form

$$
C_{t}^{\mathrm{AH}}:=\int h_{0}\left(\frac{x}{t}\right) B^{*}(t, x) B(t, x) d x
$$

which is the usual Araki-Haag detector [AH67]. Arguing as above one can justify that these detectors are sensitive only to particles whose velocities belong to the support of $h_{0}$, i.e., are in a neighbourhood of $\nabla \omega(p)$. However, one cannot conclude in this case that the masses $m^{\prime}$ of these particles are close to $m$. Thus $t \mapsto C_{t}^{\mathrm{AH}}$ is sensitive not only to neutral particles of mass $m$, but may also detect some neutral or charged particles whose mass hyperboloids are embedded in the multiparticle spectrum in the respective sector. (Charged particles with isolated mass hyperboloids can be excluded by exploiting the energy-momentum transfer of $B$, similarly as above). While this sensitivity to other particles would disappear in the next step of our analysis, which concerns products of detectors (see (1.8) below), we find it conceptually more satisfactory to work from the outset with detectors (1.4), which are only sensitive to neutral particles of mass $m$. A more technical reason to use these detectors, related to difficulty (3), will be discussed later on in this section.

Coming back to the construction of the 'charged particles free' subspace $\mathcal{H}^{\text {cpf }}$, we fix some open bounded set $\Delta \subset G_{2 m}$, which is small compared to the mass gap, (i.e.,

\footnotetext{
3 If the particle of type $q$ is neutral, we have $m_{q}=m$ since we assumed that there is only one isolated mass hyperboloid in $\mathcal{S} p U$. If the particle of type $q$ is charged, we have $m_{q} \geq m$, since otherwise the multiparticle spectrum $G_{2 m}$ in the vacuum sector would start below $E=2 m$ due to the presence of pairs of oppositely charged particles of mass $m_{q}$.
} 
s.t. $(\bar{\Delta}-\bar{\Delta}) \cap \mathcal{S} p U=\{0\})$ and let $\mathbb{1}_{\Delta}(U)$ be the corresponding spectral projection. We intend to characterize states from the range of $\mathbb{1}_{\Delta}(U)$ which are configurations of $n \geq 2$ neutral particles of mass $m$. Let us consider one such configuration consisting of particles whose energy-momentum vectors are centered around some $\tilde{p}_{i} \in H_{m}, i=1, \ldots, n$, which satisfy $\tilde{p}_{1}+\cdots+\tilde{p}_{n} \in \Delta$ and $\tilde{p}_{i} \neq \tilde{p}_{j}$ for $i \neq j$. We denote by $t \mapsto C_{i, t}$, $i=1, \ldots, n$, detectors of the form (1.4) sensitive to neutral particles whose energymomentum vectors are close to $\tilde{p}_{i}$. In particular, we require that the corresponding functions $h_{i} \in C_{0}^{\infty}\left(T^{*} \mathbb{R}^{d}\right)$ have disjoint supports in the first variable. A coincidence arrangement of this collection of detectors, defined as

$$
Q_{n}^{+}(\Delta) \Psi:=\mathrm{s}-\lim _{t \rightarrow \infty} C_{1, t} \ldots C_{n, t} \Psi, \quad \Psi \in \mathbb{1}_{\Delta}(U) \mathcal{H}
$$

is an asymptotic observable sensitive to the prescribed configuration of $n$ neutral particles. In fact, for $\Psi$ from the subspace of Haag-Ruelle scattering states, it follows from our discussion of individual detectors above that any vector from the range of $Q_{n}^{+}(\Delta)$ contains only the prescribed configuration of neutral particles. (The presence of any other particles is energetically excluded, since $Q_{n}^{+}(\Delta)$ commutes with $\mathbb{1}_{\Delta}(U)$, $\tilde{p}_{1}+\cdots+\tilde{p}_{n} \in \Delta$ and $\Delta$ is small compared to the mass gap). It is an important finding of the present paper that the same holds for any $\Psi \in \mathbb{1}_{\Delta}(U) \mathcal{H}$, including the existence of the limit in (1.8). Leaving the question of convergence in (1.8) to the later part of this Introduction, we set $\mathcal{H}(\Delta)=\mathbb{1}_{\Delta}(U) \mathcal{H}$ and define the $n$-particle component of the 'charged particles free' subspace associated with the set $\Delta$ as

$$
\mathcal{H}_{n}^{\mathrm{cpf}}(\Delta):=\operatorname{Span}\left\{Q_{n, \alpha}^{+}(\Delta) \mathcal{H}(\Delta): \alpha \in J\right\}^{\mathrm{cl}},
$$

where the span extends over the collection of all the asymptotic observables of the form (1.8), corresponding to various configurations of $n$ neutral particles with total energymomentum in $\Delta$. We show in Theorems 2.8 and 2.9 that

$$
\mathcal{H}_{n}^{\mathrm{cpf}}(\Delta)=\mathcal{H}_{n}^{+}(\Delta)
$$

where $\mathcal{H}_{n}^{+}(\Delta)$ is the subspace of $n$-particle Haag-Ruelle scattering states (of particles from $H_{m}$ ) with total energy-momentum in $\Delta$. Since the vacuum and the neutral singleparticle states are also 'charged particles free', we set

$$
\mathcal{H}^{\mathrm{cpf}}:=\mathbb{C} \Omega \oplus \mathbb{1}_{H_{m}}(U) \mathcal{H} \oplus \operatorname{Span}\left\{\mathcal{H}_{n}^{\mathrm{cpf}}(\Delta): n \geq 2, \Delta \subset G_{2 m}\right\}^{\mathrm{cl}},
$$

where the span extends over all open bounded sets $\Delta$ s.t. $(\bar{\Delta}-\bar{\Delta}) \cap \mathcal{S} p U=\{0\}{ }^{4}$ Making use of (1.10), we immediately obtain the generalized AC relation $\mathcal{H}^{\text {cpf }}=\mathcal{H}^{+}$ and criterion (1.3) for conventional AC.

A crucial technical step of our analysis is the proof of existence of the limits (1.8). We recall that the convergence of Araki-Haag detectors on the subspace of scattering states of bounded energy follows from the results in [AH67, Bu90]. However, their convergence on the orthogonal complement of this subspace, which is of great importance for the question of $\mathrm{AC}$, is a long-standing open problem, discussed for example in [Ha]. To

\footnotetext{
4 If the multiparticle spectrum $G_{2 m}$ contains an embedded mass hyperboloid $H_{m^{\prime}}, m^{\prime} \geq 2 m$, the corresponding spectral subspace belongs to the orthogonal complement of $\mathcal{H}^{\text {cpf }}$ by relation (1.10). This is conceptually not completely satisfactory, since the particles from $H_{m^{\prime}}$ are neutral. One could improve on this point by including also detectors sensitive to particles from $H_{m^{\prime}}$ and using a variant of Haag-Ruelle theory suitable for embedded mass-shells [He71,Dy05]. However, we leave this problem for future investigations.
} 
tackle this problem, we essentially reduce it to scattering theory of an $n$-body dispersive Hamiltonian. Let us explain this reduction:

Let us set $Q_{n, t}(\Delta):=C_{1, t} \ldots C_{n, t} \mathbb{1}_{\Delta}(U)$. Exploiting locality and the disjointness of supports of $h_{i}$ (in the first variable) we can write:

$Q_{n, t}(\Delta) \Psi=\int \mathrm{H}_{t}^{\mathrm{W}}(\underline{x}, \underline{y}) B_{1}^{*}\left(t, x_{1}\right) \ldots B_{n}^{*}\left(t, x_{n}\right) B_{1}\left(t, y_{1}\right) \ldots B_{n}\left(t, y_{n}\right) \Psi d \underline{x} d \underline{y}+O\left(t^{-\infty}\right)$,

where $\underline{x}:=\left(x_{1}, \ldots, x_{n}\right), \underline{y}:=\left(y_{1}, \ldots, y_{n}\right)$ and we denote by $\mathrm{H}_{t}^{\mathrm{w}}(\underline{x}, \underline{y})$ the distributional kernel of

$$
\mathrm{H}_{t}^{\mathrm{W}}:=h_{1, t}^{\mathrm{w}} \otimes \cdots \otimes h_{n, t}^{\mathrm{w}}
$$

and by $O\left(t^{-\infty}\right)$ a term which vanishes in norm faster than any inverse power of $t$.

Exploiting the fact that $\Psi \in \mathbb{1}_{\Delta}(U) \mathcal{H}$ and our assumptions on the energy-momentum transfers of $B_{i}$, we can write

$$
B_{1}\left(t, y_{1}\right) \ldots B_{n}\left(t, y_{n}\right) \Psi=\Omega\left(\Omega \mid B_{1}\left(t, y_{1}\right) \ldots B_{n}\left(t, y_{n}\right) \Psi\right)_{\mathcal{H}}
$$

We set

$$
F_{t}(\underline{y}):=\left(\Omega \mid B_{1}\left(t, y_{1}\right) \ldots B_{n}\left(t, y_{n}\right) \Psi\right)_{\mathcal{H}},
$$

and note that by a result from [Bu90], $F_{t} \in L^{2}\left(\mathbb{R}^{n d}\right)$ for any $t \in \mathbb{R}$. Thus we obtain from (1.12):

$$
Q_{n, t}(\Delta) \Psi=\int\left(\int \mathrm{H}_{t}^{\mathrm{W}}(\underline{x}, \underline{y}) F_{t}(\underline{y}) d \underline{y}\right) B_{1}^{*}\left(t, x_{1}\right) \ldots B_{n}^{*}\left(t, x_{n}\right) \Omega d \underline{x}+O\left(t^{-\infty}\right) .
$$

If we replaced the expression in brackets above by a sum of products of $n$ positive energy solutions of the Klein-Gordon equation, the first term on the r.h.s. of (1.15) would become an $n$-particle scattering state approximant. While such a substitution is not possible at finite times, it can be performed asymptotically: In fact, as we show in Theorem 5.4, there exists the limit

$$
F_{+}=\lim _{t \rightarrow \infty} \mathrm{e}^{\mathrm{i} t \tilde{\omega}\left(D_{\underline{x}}\right)} \mathrm{H}_{t}^{\mathrm{w}} F_{t},
$$

where $\tilde{\omega}\left(D_{\underline{x}}\right):=\omega\left(D_{x_{1}}\right)+\cdots+\omega\left(D_{x_{n}}\right)$. In Theorem 4.1 we verify that the existence of this limit implies the convergence of $t \mapsto Q_{n, t}(\Delta) \Psi$ as $t \rightarrow \infty$. The key step towards the proof of convergence in (1.16), which we take in Lemma 4.2, is to show that $F_{t}$ satisfies the following evolution equation with a source term:

$$
\partial_{t} F_{t}=-\mathrm{i} \tilde{\omega}\left(D_{\underline{x}}\right) F_{t}+R_{t},
$$

where the source term satisfies $\mathrm{H}_{t}^{\mathrm{w}} R_{t}=O\left(t^{-\infty}\right)$ due to locality and the disjointness of supports of $h_{i}$ in the first argument.

It is easy to see that the Schrödinger equation of a system of massive particles with relativistic dispersion relations, interacting with a rapidly decaying potential, has a general form of (1.17). Thus we reduced the problem of convergence of the generalized ArakiHaag detectors in (1.8) to the question of existence of the limit (1.16) in a dispersive system described by the evolution equation (1.17). 
For $n=2$ we solved this problem in a recent publication [DG12], for standard ArakiHaag detectors whose symbols $h_{i}$ are independent of momentum, following the approach of Graf [Gr90]: we combined a large velocity propagation estimate, which in our context says that particles cannot move faster than light, with a phase space propagation estimate, which encodes the fact that the instantaneous velocity of a particle equals its average velocity at large times. The convex Graf function, appearing in the derivation of this latter estimate, must vanish near the collision plane $\left\{x_{1}=x_{2}\right\}$ to ensure a rapid decay of the rest term $R_{t}$ in (1.17). Due to this restriction, the method does not generalize to the case $n>2$, which involves several collision planes $\left(\left\{x_{1}=x_{2}\right\},\left\{x_{1}=x_{3}\right\},\left\{x_{2}=x_{3}\right\}\right.$, etc.) In fact, since the Graf function is convex, it would have to vanish in the convex hull of these collision planes, which contains the relevant part of the configuration space. This difficulty is one of several obstacles which hinder our understanding of AC for dispersive systems of three or more particles [Zi97, Ge91].

A solution of this problem in the case of a product of $n \geq 3$ particle detectors is the main technical result of the present paper. In this case it is instrumental to use symbols $h_{i}$ in (1.4) which depend also on momentum. As we mentioned above, they have the form $h_{i}(x, \xi)=h_{0, i}(x) \chi(x-\nabla \omega(\xi))$, where the supports of $h_{0, i} \in C_{0}^{\infty}\left(\mathbb{R}^{d}\right)$ are disjoint (with some minimal distance $\varepsilon>0$ ) and $\chi$ is supported in a ball around zero whose radius is $\varepsilon^{\prime} \ll \varepsilon$. For such symbols $h_{i}$ and $H_{t}^{\mathrm{w}}$ as in (1.13) we prove in Proposition 5.3 the following new variant of a phase space propagation estimate:

$$
\int_{1}^{+\infty}\left\|\left(\underline{x} / t-\nabla \tilde{\omega}\left(D_{\underline{x}}\right)\right) \cdot H_{t}^{\mathrm{w}} F_{t}\right\|^{2} \frac{d t}{t}<\infty .
$$

Abstract arguments, which are an extension of results of standard scattering theory to inhomogeneous evolution equations like (1.17), allow then to deduce from (1.18) the existence of the limit (1.16).

Our paper is organized as follows: In Sect. 2 we recall the framework of algebraic QFT, introduce some central concepts and state our main results. Section 3 contains more technical preliminaries. In Sect. 4 we show that the existence of the intermediate limit (1.16) implies the convergence of the approximating sequences of detectors in (1.8). Section 5 contains the proof of existence of the intermediate limit (1.16). In Sect. 6 we show that the ranges of the asymptotic observables (1.8) span the entire subspace of the Haag-Ruelle scattering states.

This paper can be seen as a (non-trivial) generalization of our work [DG12] on twoparticle scattering to the $n$-particle case. Readers who are familiar with [DG12] will find material which is special to the $n>2$ case in Sects. 2.3-3.2, 3.5, 3.6 and in Sect. 5.

\section{Framework and Results}

In this section we recall the Haag-Kastler framework of local quantum field theory and state our main results. The preliminary Sects. 2.1 and 2.2 are similar to the corresponding subsections of [DG12].

\subsection{Nets of local observables. We base our theory on a net}

$$
\mathcal{O} \mapsto \mathfrak{A}(\mathcal{O}) \subset B(\mathcal{H})
$$

of von Neumann algebras attached to open bounded regions of Minkowski spacetime $\mathbb{R}^{1+d}$, which satisfies the assumptions of isotony, locality, covariance w.r.t. translations, positivity of energy, uniqueness of the vacuum and cyclicity of the vacuum. 
Isotony says that $\mathfrak{A}\left(\mathcal{O}_{1}\right) \subset \mathfrak{A}\left(\mathcal{O}_{2}\right)$ if $\mathcal{O}_{1} \subset \mathcal{O}_{2}$, which allows to define the $C^{*}$ inductive limit of the net, denoted by $\mathfrak{A}$. Locality requires that $\mathfrak{A}\left(\mathcal{O}_{1}\right) \subset \mathfrak{A}\left(\mathcal{O}_{2}\right)^{\prime}$ if $\mathcal{O}_{1}$ and $\mathcal{O}_{2}$ are spacelike separated. To state the remaining postulates, we introduce a strongly continuous unitary representation of translations

$$
\mathbb{R}^{1+d} \ni(t, x) \mapsto U(t, x)=: \mathrm{e}^{\mathrm{i}(t H-x \cdot P)} \quad \text { on } \mathcal{H},
$$

which induces a group of automorphisms of $\mathfrak{A}$ :

$$
\alpha_{t, x}(B):=B(t, x):=U(t, x) B U(t, x)^{*}, \quad B \in \mathfrak{A},(t, x) \in \mathbb{R}^{1+d} .
$$

Covariance requires that

$$
\alpha_{t, x}(\mathfrak{A}(\mathcal{O}))=\mathfrak{A}(\mathcal{O}+(t, x)), \forall \text { open bounded } \mathcal{O} \text { and }(t, x) \in \mathbb{R}^{1+d}
$$

We will need a restrictive formulation of positivity of energy, suitable for massive theories. We denote by $H_{m}:=\left\{(E, p) \in \mathbb{R}^{1+d}: E=\sqrt{p^{2}+m^{2}}\right\}$ the mass hyperboloid of a particle of mass $m>0$ and set $G_{\mu}:=\left\{(E, p) \in \mathbb{R}^{1+d}: E \geq \sqrt{p^{2}+\mu^{2}}\right\}$. We assume that:

$$
\begin{aligned}
& \text { (i) } \mathcal{S}_{p} U=\{0\} \cup H_{m} \cup G_{2 m}, \\
& \text { (ii) } \mathbb{1}_{\{0\}}(U)=|\Omega\rangle\langle\Omega|, \Omega \text { cyclic for } \mathfrak{A} .
\end{aligned}
$$

Here we denoted by $\mathcal{S} p U \subset \mathbb{R}^{1+d}$ the spectrum of $(H, P)$ and by $\mathbb{1}_{\Delta}(U)$ the spectral projection on a Borel set $\Delta \subset \mathbb{R}^{1+d}$. The unit vector $\Omega$ will be called the vacuum vector. Part (i) in (2.2) encodes positivity of energy and the presence of an upper and lower mass gap $m$. Part (ii) covers the uniqueness and cyclicity of the vacuum.

Remark 2.1. We adopt the restrictive form of the spectrum condition (2.2) (i) to remain consistent with the discussion of AC in the Introduction. We remark, however, that our main results, Theorems 2.8 and 2.9 below, remain valid as they stand if the assumption (2.2) (i) is relaxed to $\mathcal{S} p U=\{0\} \cup H_{m} \cup \tilde{G}, \tilde{G} \subset G_{\mu}, m<\mu \leq 2 m$. If $\tilde{G} \backslash G_{2 m}$ consists of isolated mass hyperboloids, our results can easily be modified so as to take the additional types of neutral particles into account.

2.2. Relevant classes of observables. In this subsection we introduce some classes of observables, which are important for our discussion. We start with the definition of almost local operators. We denote by $\mathcal{O}_{r}:=\left\{(t, x) \in \mathbb{R}^{1+d}:|t|+|x|<r\right\}$ the double cone of radius $r$ centered at 0 .

Definition 2.2. $B \in \mathfrak{A}$ is almost local if there exists a family $A_{r} \in \mathfrak{A}\left(\mathcal{O}_{r}\right)$ s.t. $\left\|B-A_{r}\right\| \in$ $O\left(\langle r\rangle^{-\infty}\right)$. $\left(\right.$ Here $\left.\langle r\rangle:=\sqrt{1+r^{2}}\right)$.

For $B \in \mathfrak{A}$, we denote by $\widehat{B}$ the Fourier transform of $(t, x) \mapsto B(t, x)$ defined as an operator-valued distribution:

$$
\widehat{B}(E, p):=(2 \pi)^{-(1+d) / 2} \int \mathrm{e}^{-\mathrm{i}(E t-p \cdot x)} B(t, x) d t d x .
$$


The support of $\widehat{B}$, denoted by $\operatorname{supp}(\widehat{B}) \subset \mathbb{R}^{1+d}$, is called the energy-momentum transfer of $B$. We recall the following well-known properties:

$$
\begin{aligned}
& \text { (i) } \widehat{\alpha_{t, x}(B)}(E, p)=\mathrm{e}^{\mathrm{i}(E t-p \cdot x)} \widehat{B}(E, p) \\
& \text { (ii) } \operatorname{supp}\left(\widehat{B^{*}}\right)=-\operatorname{supp}(\widehat{B}) \\
& \text { (iii) } B \mathbb{1}_{\Delta}(U)=\mathbb{1} \frac{}{\Delta+\operatorname{supp}(\widehat{B})}(U) B \mathbb{1}_{\Delta}(U), \forall \text { Borel sets } \Delta \subset \mathbb{R}^{1+d} .
\end{aligned}
$$

For (iii) we refer to [Ar82, Theorem 5.3]. Now we are ready to define another important class of observables, which are the energy decreasing operators:

Definition 2.3. $B \in \mathfrak{A}$ is energy decreasing if $\operatorname{supp}(\widehat{B}) \cap V_{+}=\emptyset$, where $V_{+}:=\{(E, p)$ : $E \geq|p|\}$ is the closed forward light cone.

In the rest of the paper we will work with the following set of observables:

Definition 2.4. We denote by $\mathcal{L}_{0} \subset \mathfrak{A}$ the subspace spanned by the elements $B \in \mathfrak{A}$ such that:
(i) $B$ is energy decreasing, $\operatorname{supp}(\widehat{B})$ is compact,
(ii) $\mathbb{R}^{1+d} \ni(t, x) \mapsto B(t, x) \in \mathfrak{A}$ is $C^{\infty}$ in norm,
(iii) $\partial_{t, x}^{\alpha} B(t, x)$ is almost local for all $\alpha \in \mathbb{N}^{1+d}$.

Clearly, if (i) and (ii) hold, then $\partial_{t, x}^{\alpha} B(t, x)$ is energy decreasing for any $\alpha \in \mathbb{N}^{1+d}$. It is easy to give examples of elements of $\mathcal{L}_{0}$ : let $A \in \mathfrak{A}(\mathcal{O})$ and $f \in \mathcal{S}\left(\mathbb{R}^{1+d}\right)$ with supp $\widehat{f}$ compact and supp $\widehat{f} \cap V_{+}=\emptyset$. Then

$$
B=(2 \pi)^{-(1+d) / 2} \int f(t, x) A(t, x) d t d x
$$

belongs to $\mathcal{L}_{0}$, since $\widehat{B}(E, p)=\widehat{f}(E, p) \widehat{A}(E, p)$. (See (3.1) below for definition of $\left.\widehat{f}\right)$.

2.3. Pseudo-differential operators. We consider the phase space $T^{*} \mathbb{R}^{\ell}=\mathbb{R}^{\ell} \times\left(\mathbb{R}^{\ell}\right)^{\prime}$, whose elements are denoted by $(x, \xi)$. For $h \in \mathcal{S}\left(T^{*} \mathbb{R}^{\ell}\right)$ we define its Weyl quantization $h^{\mathrm{w}}$ by

$$
h^{\mathrm{w}} u(x)=(2 \pi)^{-\ell} \int \mathrm{e}^{\mathrm{i}(x-y) \cdot \xi} h\left(\frac{x+y}{2}, \xi\right) u(y) d y d \xi, \quad u \in \mathcal{S}\left(\mathbb{R}^{\ell}\right) .
$$

It is well known that $h^{\mathrm{w}}$ is bounded on $\mathcal{S}\left(\mathbb{R}^{\ell}\right)$ and $L^{2}\left(\mathbb{R}^{\ell}\right)$.

Denoting by $A(x, y) \in \mathcal{S}^{\prime}\left(\mathbb{R}^{\ell} \times \mathbb{R}^{\ell}\right)$ the distributional kernel of $A: \mathcal{S}\left(\mathbb{R}^{\ell}\right) \rightarrow \mathcal{S}^{\prime}\left(\mathbb{R}^{\ell}\right)$, one has:

$$
h^{\mathrm{w}}(x, y)=(2 \pi)^{-\ell / 2} \breve{h}\left(\frac{x+y}{2}, x-y\right)
$$

where $\breve{h}(x, y)=(2 \pi)^{-\ell / 2} \int \mathrm{e}^{\mathrm{i} y \cdot \xi} h(x, \xi) d \xi$ is the inverse Fourier transform of $h$ in the $\xi$ variable.

We refer to [Ho85] and [DG97, Appendix D] for systematic expositions of the Weyl quantization. Properties needed in the present work are summarized in Sect. 3.2 below. 
2.4. Results. To $B \in \mathcal{L}_{0}, h \in \mathcal{S}\left(T^{*} \mathbb{R}^{d}\right)$, we associate the one-particle detector:

$$
\begin{aligned}
C_{t} & :=\int B^{*}(t, x) h_{t}^{\mathrm{w}}(x, y) B(t, y) d x d y \\
& =\int B^{*}\left(t, x+\frac{y}{2}\right) \breve{h}\left(\frac{x}{t}, y\right) B\left(t, x-\frac{y}{2}\right) d x d y,
\end{aligned}
$$

where we set $h_{t}(x, \xi)=h\left(\frac{x}{t}, \xi\right)$. In view of Lemma 3.4 below, one has

$$
\sup _{t \in \mathbb{R}}\left\|C_{t} \mathbb{1}_{\tilde{\Delta}}(U)\right\|<\infty
$$

for any bounded Borel set $\tilde{\Delta}$.

A much more convenient formula for $C_{t}$, using notation introduced below in Sect. 3, is:

$$
C_{t}=\mathrm{e}^{\mathrm{i} t H}\left(a_{B}^{*} \circ\left(\mathbb{1}_{\mathcal{H}} \otimes h_{t}^{\mathrm{w}}\right) \circ a_{B}\right) \mathrm{e}^{-\mathrm{i} t H}
$$

In particular, it remains meaningful if $h_{t}^{\mathrm{w}}$ is replaced by any bounded operator on $L^{2}\left(\mathbb{R}^{d}\right)$. For example, for symbols $h_{0} \in C_{0}^{\infty}\left(\mathbb{R}^{d}\right)$, independent of momentum, we recover from (2.9) the conventional Araki-Haag detectors from [AH67]:

$$
C_{t}^{\mathrm{AH}}:=\int B^{*}(t, x) h_{0}\left(\frac{x}{t}\right) B(t, x) d x,
$$

which we considered in [DG12]. These detectors are only sensitive to average velocity $x / t$ of a particle. In view of formula (2.8), our detectors $C_{t}$ are essentially averages (w.r.t. $y$ ) of the conventional Araki-Haag detectors, and are also sensitive to momentum $\xi$ of a particle.

We fix now $B_{i} \in \mathcal{L}_{0}, h_{i} \in \mathcal{S}\left(T^{*} \mathbb{R}^{d}\right)$ for $1 \leq i \leq n$ and define $C_{i, t}$ as in (2.8). For any open bounded subset $\Delta \subset G_{2 m}$ we define the $n$-particle detector:

$$
Q_{n, t}(\Delta):=C_{1, t} \ldots C_{n, t} \mathbb{1}_{\Delta}(U) .
$$

Our main technical result is the strong convergence of $Q_{n, t}(\Delta)$ as $t \rightarrow \infty$ if the extension of $\Delta$ is smaller than the mass gap (i.e., $(\bar{\Delta}-\bar{\Delta}) \cap \mathcal{S} p U=\{0\}), B$ is $\Delta$-admissible in the sense of Definition 2.5 and $\mathrm{H}:=h_{1} \otimes \cdots \otimes h_{n}$ is admissible in the sense of Definition 2.7.

Definition 2.5. Let $\Delta \subset \mathbb{R}^{1+d}$ be an open bounded set and $B_{1}, \ldots, B_{n} \in \mathcal{L}_{0}$. We say that $\underline{B}=\left(B_{1}, \ldots, B_{n}\right)$ is $\Delta$-admissible if

$$
\begin{gathered}
\left(-\operatorname{supp}\left(\widehat{B}_{i}\right)\right) \cap \mathcal{S} p U \subset H_{m}, \quad i=1, \ldots, n, \\
\quad-\left(\operatorname{supp}\left(\widehat{B}_{1}\right)+\cdots+\operatorname{supp}\left(\widehat{B}_{n}\right)\right) \subset \Delta, \\
\left(\bar{\Delta}+\operatorname{supp}\left(\widehat{B}_{1}\right)+\cdots+\operatorname{supp}\left(\widehat{B}_{n}\right)\right) \cap \mathcal{S} p U \subset\{0\} .
\end{gathered}
$$

Remark 2.6. In Lemma 7.4 it is shown that if $\Delta \subset G_{2 m}$ is an open bounded set s.t. $(\bar{\Delta}-\bar{\Delta}) \cap \mathcal{S} p U \subset\{0\}$ and $-\operatorname{supp}\left(\widehat{B}_{1}\right), \ldots,-\operatorname{supp}\left(\widehat{B}_{n}\right)$ are sufficiently small neighbourhoods of vectors $\tilde{p}_{1}, \ldots, \tilde{p}_{n} \in H_{m}$ s.t. $\tilde{p}_{i} \neq \tilde{p}_{j}$ for $i \neq j$ and $\tilde{p}_{1}+\cdots+\tilde{p}_{n} \in \Delta$ then $\underline{B}=\left(B_{1}, \ldots, B_{n}\right)$ is $\Delta$-admissible. We also note that for such $\Delta$ (2.11) and (2.12) cannot be simultaneously satisfied for $n=1$ if $B_{1} \neq 0$. 
Let us introduce the notation

$$
\begin{aligned}
D_{0} & :=\left\{\underline{x} \in \mathbb{R}^{n d}: x_{i}=x_{j} \text { for some } i \neq j\right\}, \\
\mathcal{B}(0, \epsilon) & :=\left\{\underline{x} \in \mathbb{R}^{n d}:|\underline{x}|<\epsilon\right\}, \\
\tilde{\mathcal{B}}(0, \epsilon) & :=\left\{\underline{x} \in \mathbb{R}^{n d}:\left|x_{i}\right|<\epsilon, i=1, \ldots, n\right\},
\end{aligned}
$$

and define $\tilde{\omega}(\xi):=\omega\left(\xi_{1}\right)+\cdots+\omega\left(\xi_{n}\right)$. Note that $\nabla \tilde{\omega}(\xi) \in \tilde{\mathcal{B}}(0,1)$ for any $\xi \in \mathbb{R}^{n d}$.

Definition 2.7. Let $\mathrm{H} \in C_{0}^{\infty}\left(T^{*} \mathbb{R}^{n d} ; \mathbb{R}^{\ell}\right)$. We say that $\mathrm{H}$ is admissible, if there exists a compact set $K \subset \mathbb{R}^{\text {nd }}$ and $\epsilon>0$ such that $K+\tilde{\mathcal{B}}(0, \epsilon) \subset \tilde{\mathcal{B}}(0,1) \backslash D_{0}$ and

$$
\operatorname{supp} \mathrm{H} \subset\left\{(\underline{x}, \underline{\xi}) \in T^{*} \mathbb{R}^{n d}: \nabla \tilde{\omega}(\underline{\xi}) \in K, \underline{x}-\nabla \tilde{\omega}(\underline{\xi}) \in \mathcal{B}(0, \epsilon)\right\} .
$$

Theorem 2.8. Let $\Delta \subset G_{2 m}$ be an open bounded set s.t. $(\bar{\Delta}-\bar{\Delta}) \cap \mathcal{S} p U=\{0\}$. Let $\underline{B}=\left(B_{1}, \ldots, B_{n}\right)$ be a collection of elements of $\mathcal{L}_{0}$ s.t. $\underline{B}$ is $\Delta$-admissible and let $\underline{h}=\left(h_{1}, \ldots, h_{n}\right)$ be a collection of elements of $C_{0}^{\infty}\left(T^{*} \mathbb{R}^{d}\right)$ s.t. $\mathrm{H}=h_{1} \otimes \cdots \otimes h_{n}$ is admissible. Then there exists the limit

$$
Q_{n}^{+}(\Delta):=\mathrm{s}-\lim _{t \rightarrow \infty} C_{1, t} \ldots C_{n, t} \mathbb{1}_{\Delta}(U),
$$

where $C_{i, t}$ are defined in (2.8) for $B_{i}, h_{i}, i=1, \ldots, n$. The range of $Q_{n}^{+}(\Delta)$ belongs to $\mathcal{H}_{n}^{+}(\Delta):=\mathbb{1}_{\Delta}(U) \mathcal{H}_{n}^{+}$, where $\mathcal{H}_{n}^{+}$is the subspace of $n$-particle scattering states. (See Definition 6.7).

Proof. Follows immediately from Theorems 4.1 and 5.4.

Theorem 2.8 substantiates our discussion below formula (1.8) in the Introduction, where we argued that vectors from the ranges of $Q_{n}^{+}(\Delta)$ should describe configurations of $n$ neutral particles with total energies-momenta in $\Delta$. This theorem allows us to define the $n$-particle component of the 'charged particles free' subspace $\mathcal{H}_{n}^{\mathrm{cpf}}(\Delta)$ associated with any open bounded set $\Delta \subset G_{2 m}$ s.t. $(\bar{\Delta}-\bar{\Delta}) \cap \mathcal{S} p U=\{0\}$ :

$$
\mathcal{H}_{n}^{\mathrm{cpf}}(\Delta):=\operatorname{Span}\left\{Q_{n, \alpha}^{+}(\Delta) \mathcal{H}(\Delta): \alpha \in J\right\}^{\mathrm{cl}},
$$

where $J$ is the collection of pairs $\alpha=(\underline{B}, \underline{h})$ satisfying the conditions from Theorem 2.8 and $Q_{n, \alpha}^{+}(\Delta)$ is the limit (2.18) corresponding to $\alpha$. We also define the total 'charged particles free' subspace:

$$
\mathcal{H}^{\mathrm{cpf}}:=\mathbb{C} \Omega \oplus \mathbb{1}_{H_{m}}(U) \mathcal{H} \oplus \operatorname{Span}\left\{\mathcal{H}_{n}^{\mathrm{cpf}}(\Delta): n \geq 2, \Delta \subset G_{2 m}\right\}^{\mathrm{cl}},
$$

where the span extends over all open bounded sets $\Delta \subset G_{2 m}$ s.t. $(\bar{\Delta}-\bar{\Delta}) \cap \mathcal{S} p U=\{0\}$. It follows immediately from Theorem 2.8 that

$$
\mathcal{H}_{n}^{\text {cpf }}(\Delta) \subset \mathcal{H}_{n}^{+}(\Delta) \text { and therefore } \mathcal{H}^{\text {cpf }} \subset \mathcal{H}^{+},
$$

where $\mathcal{H}^{+}$is the subspace of all scattering states of neutral particles of mass $m$ (see Definition 6.7). The last inclusion can be interpreted as a weak variant of AC, as it says that certain subspace $\mathcal{H}^{\text {cpf }} \subset \mathcal{H}$, defined without reference to scattering states, is in fact contained in $\mathcal{H}^{+}$. The larger the subspace $\mathcal{H}^{\mathrm{cpf}}$ is, the closer we are to verifying AC proper. For example, if we could show that $\mathcal{H}^{\text {cpf }}=\mathcal{H}$, conventional AC would follow, which gives one implication in our criterion for AC stated in (1.3). The opposite implication is given by the following theorem, which shows that the inclusions in (2.21) are in fact equalities. This result, whose proof is given in Sect. 7, guarantees, in particular, that $\mathcal{H}_{n}^{\mathrm{cpf}}(\Delta) \neq 0$ for any $\Delta$ as specified above. 
Theorem 2.9. Let $\Delta \subset G_{2 m}$ be an open bounded set such that $(\bar{\Delta}-\bar{\Delta}) \cap \mathcal{S} p U=\{0\}$. Let $\mathcal{H}_{n}^{\mathrm{cpf}}(\Delta)$ and $\mathcal{H}^{\mathrm{cpf}}$ be as defined in (2.19) and (2.20), respectively. Then

$$
\mathcal{H}_{n}^{\mathrm{cpf}}(\Delta)=\mathcal{H}_{n}^{+}(\Delta) \text { and therefore } \mathcal{H}^{\mathrm{cpf}}=\mathcal{H}^{+},
$$

where $\mathcal{H}_{n}^{+}(\Delta):=\mathbb{1}_{\Delta}(U) \mathcal{H}_{n}^{+}, \mathcal{H}_{n}^{+}$is the subspace of $n$-particle scattering states and $\mathcal{H}^{+}$ is the subspace of all scattering states. (See Definition 6.7).

\section{Preliminaries}

In this section we specify our notation and collect some basic properties of particle detectors. Sections 3.3, 3.4 are similar to [DG12, Subsect. 3.2, 3.3]. The remaining subsections contain essential generalizations of the material from [DG12].

\subsection{Notation.}

- By $x, x_{1}, x_{2}, \ldots$ we denote elements of $\mathbb{R}^{d}$ and by $\xi, \xi_{1}, \xi_{2}, \ldots$ elements of $\left(\mathbb{R}^{d}\right)^{\prime}$. We write $T^{*} \mathbb{R}^{d}:=\mathbb{R}^{d} \times\left(\mathbb{R}^{d}\right)^{\prime}$ to denote the phase space.

- We set $\underline{x}=\left(x_{1}, \ldots, x_{n}\right)$ and $\xi=\left(\xi_{1}, \ldots, \xi_{n}\right)$ to denote elements of $\mathbb{R}^{n d}$ and $\left(\mathbb{R}^{n d}\right)^{\prime}$. The Lebesgue measure on $\mathbb{R}^{\bar{n} d}$ is denoted $d \underline{x}$.

- We write $K \Subset \mathbb{R}^{\ell}$ if $K$ is a compact subset of $\mathbb{R}^{\ell}$.

- We set $\langle x\rangle:=\left(1+x^{2}\right)^{\frac{1}{2}}$ for $x \in \mathbb{R}^{d}$ and $\omega(p)=\left(p^{2}+m^{2}\right)^{\frac{1}{2}}$ for $p \in \mathbb{R}^{d}$.

- We denote the momentum operator $\mathrm{i}^{-1} \nabla_{x}$ by $D_{x}$.

- We denote by $(t, x)$ or $(E, p)$ elements of $\mathbb{R}^{1+d}$.

- If $f: \mathbb{R}^{1+d} \rightarrow \mathbb{C}$ we will denote by $f_{t}: \mathbb{R}^{d} \rightarrow \mathbb{C}$ the function $f_{t}(\cdot):=f(t, \cdot)$.

- We denote by $\mathcal{S}\left(\mathbb{R}^{1+d}\right)$ the Schwartz class in $\mathbb{R}^{1+d}$. If $f \in \mathcal{S}\left(\mathbb{R}^{1+d}\right)$ we define its (unitary) Fourier transform:

$$
\begin{aligned}
\widehat{f}(E, p) & :=(2 \pi)^{-(1+d) / 2} \int \mathrm{e}^{\mathrm{i}(E t-p \cdot x)} f(t, x) d t d x, \\
\widetilde{f}(t, x) & :=(2 \pi)^{-(1+d) / 2} \int \mathrm{e}^{-\mathrm{i}(E t-p \cdot x)} f(E, p) d E d p .
\end{aligned}
$$

(Note the different sign in the exponent in comparison with (2.3), where the Fourier transform is taken in the sense of operator valued distributions).

- If $f \in \mathcal{S}\left(\mathbb{R}^{d}\right)$ we set, consistently with (3.1),

$$
\begin{aligned}
& \widehat{f}(p):=(2 \pi)^{-d / 2} \int \mathrm{e}^{-\mathrm{i} p \cdot x} f(x) d x, \\
& \check{f}(x):=(2 \pi)^{-d / 2} \int \mathrm{e}^{\mathrm{i} p \cdot x} f(p) d p .
\end{aligned}
$$

- If $h \in \mathcal{S}\left(T^{*} \mathbb{R}^{d}\right)$ is a symbol, $\widehat{h}$ and $\widetilde{h}$ denote the Fourier transform and the inverse Fourier transform w.r.t. the momentum variable $\xi$ only.

- By $\pi_{x}: T^{*} \mathbb{R}^{d} \rightarrow \mathbb{R}^{d}$ we will denote the projection from the phase space to configuration space.

- If $B$ is an observable, we write $B^{(*)}$ to denote either $B$ or $B^{*}$. We will also set

$$
B_{t}:=B(t, 0), \quad B(x):=B(0, x) \text { so that } B(t, x)=B_{t}(x) .
$$


3.2. Pseudodifferential calculus. For future reference, we recall the following wellknown facts:

Proposition 3.1. Let $h \in \mathcal{S}\left(T^{*} \mathbb{R}^{\ell}\right), h_{t}(x, \xi):=h(x / t, \xi)$ and $\omega \in C^{\infty}\left(\mathbb{R}^{\ell}\right)$ such that $\partial_{\xi}^{\alpha} \omega$ is bounded for all $|\alpha| \geq 1$. Then

(1) If $h \geq 0$ then $h_{t}^{\mathrm{w}} \geq-\frac{c}{t}$ for some $c \geq 0$ independent of $t$.

(2) $\mathrm{s}-\lim _{t \rightarrow \infty} \mathrm{e}^{\mathrm{i} t \omega\left(D_{x}\right)} h_{t}^{\mathrm{w}} \mathrm{e}^{-\mathrm{i} t \omega\left(D_{x}\right)}=h\left(\nabla \omega\left(D_{x}\right), D_{x}\right)=h(\nabla \omega(\cdot), \cdot)^{\mathrm{w}}$.

(3) $\left[\omega\left(D_{x}\right), \mathrm{i} h_{t}^{\mathrm{W}}\right]$, defined as a continuous linear map $\mathcal{S}\left(\mathbb{R}^{\ell}\right) \rightarrow \mathcal{S}\left(\mathbb{R}^{\ell}\right)$, extends to a bounded operator on $L^{2}\left(\mathbb{R}^{\ell}\right)$ s.t.:

$$
\left[\omega\left(D_{x}\right), \mathrm{i} h_{t}^{\mathrm{w}}\right]=\frac{1}{t}\left(\nabla_{\xi} \omega \cdot\left(\nabla_{x} h\right)_{t}\right)^{\mathrm{w}}+O\left(t^{-2}\right) .
$$

(4) Let $f \in C^{\infty}\left(\mathbb{R}^{\ell}\right)$ be bounded by a fixed polynomial, together with all its derivatives. Then $f(x / t) h_{t}^{\mathrm{w}}$ and $f\left(D_{x}\right) h_{t}^{\mathrm{w}}$, defined as continuous linear maps $\mathcal{S}\left(\mathbb{R}^{\ell}\right) \rightarrow \mathcal{S}\left(\mathbb{R}^{\ell}\right)$, extend to bounded operators on $L^{2}\left(\mathbb{R}^{\ell}\right)$ s.t.

$$
f(x / t) h_{t}^{\mathrm{w}}=\left(h_{1, t}\right)^{\mathrm{w}}+O\left(t^{-1}\right), \quad f\left(D_{x}\right) h_{t}^{\mathrm{w}}=h_{2, t}^{\mathrm{w}}+O\left(t^{-1}\right),
$$

where $h_{1, t}(x, \xi):=f(x / t) h(x / t, \xi)$ and $h_{2, t}(x, \xi)=f(\xi) h(x / t, \xi)$.

(5) Let $g \in \mathcal{S}\left(T^{*} \mathbb{R}^{\ell}\right)$ and $g_{t}(x, \xi)=g(x / t$, $\xi)$. Then $\left(g_{t}^{\mathrm{w}}\right)\left(h_{t}^{\mathrm{w}}\right)=\left(g_{t} h_{t}\right)^{\mathrm{W}}+O\left(t^{-1}\right)$.

(6) Let $\chi \in C_{0}^{\infty}\left(\mathbb{R}^{\ell}\right)$ be s.t. $\chi(x)=1$ near $\pi_{x} \operatorname{supp} h$. Let $\chi_{t}(x)=\chi(x / t)$ and denote the corresponding operator on $L^{2}\left(\mathbb{R}^{\ell}\right)$ also by $\chi_{t}$. Then $\left(1-\chi_{t}\right) h_{t}^{\mathrm{w}}=O\left(t^{-\infty}\right)$.

3.3. Auxiliary maps $\mathbf{a}_{\mathbf{B}}$. For $B \in \mathfrak{A}, f \in \mathcal{S}\left(\mathbb{R}^{d}\right)$ we write:

$$
B(f):=\int B(x) f(x) d x,
$$

so that $B^{*}(f)=B(\bar{f})^{*}$. If $B_{1}, B_{2} \in \mathfrak{A}$ are almost local, then

$$
\left\|\left[B_{1}\left(x_{1}\right), B_{2}\left(x_{2}\right)\right]\right\| \leq C_{N}\left\langle x_{1}-x_{2}\right\rangle^{-N}, \quad \forall N \in \mathbb{N},
$$

and consequently

$$
\left\|\left[B_{1}\left(f_{1}\right), B_{2}\left(f_{2}\right)\right]\right\| \leq C_{N} \int\left|f_{1}\left(x_{1}\right)\right|\left\langle x_{1}-x_{2}\right\rangle^{-N}\left|f_{2}\left(x_{2}\right)\right| d x_{1} d x_{2}, \quad f_{1}, f_{2} \in \mathcal{S}\left(\mathbb{R}^{d}\right) .
$$

Now we introduce auxiliary maps which will be often used in our investigation:

Definition 3.2. Let $B \in \mathfrak{A}$. We denote by $a_{B}: \mathcal{H} \rightarrow \mathcal{S}^{\prime}\left(\mathbb{R}^{d} ; \mathcal{H}\right)$ the linear operator defined as:

$$
a_{B} \Psi(x):=B(x) \Psi, \quad x \in \mathbb{R}^{d} .
$$

The operator $a_{B}: \mathcal{H} \rightarrow \mathcal{S}^{\prime}\left(\mathbb{R}^{d} ; \mathcal{H}\right)$ is continuous and

$$
B(f)=\left(\mathbb{1}_{\mathcal{H}} \otimes\langle\bar{f}|\right) \circ a_{B}, f \in \mathcal{S}\left(\mathbb{R}^{d}\right),
$$

where $\left(\mathbb{1}_{\mathcal{H}} \otimes\langle\bar{f}|\right): \mathcal{S}^{\prime}\left(\mathbb{R}^{d} ; \mathcal{H}\right) \rightarrow \mathcal{H}$ is defined on simple tensors by

$$
\left(\mathbb{1}_{\mathcal{H}} \otimes\langle\bar{f}|\right)(\Psi \otimes T)=T(f) \Psi, \quad \Psi \in \mathcal{H}, T \in \mathcal{S}^{\prime}\left(\mathbb{R}^{d}\right) .
$$


By duality $a_{B}^{*}: \mathcal{S}\left(\mathbb{R}^{d} ; \mathcal{H}\right) \rightarrow \mathcal{H}$ is continuous and we have

$$
B^{*}(f)=a_{B}^{*} \circ\left(\mathbb{1}_{\mathcal{H}} \otimes|f\rangle\right), \quad f \in \mathcal{S}\left(\mathbb{R}^{d}\right) .
$$

The group of space translations

$$
\tau_{y} \Psi(x):=\Psi(x-y), \quad y \in \mathbb{R}^{d},
$$

is strongly continuous on $\mathcal{S}^{\prime}\left(\mathbb{R}^{d} ; \mathcal{H}\right)$, and its generator is $D_{x}$ i.e., $\tau_{y}=\mathrm{e}^{-\mathrm{i} y \cdot D_{x}}$. Clearly, we have the identity:

$$
a_{B} \circ \mathrm{e}^{-\mathrm{i} y \cdot P}=\mathrm{e}^{-\mathrm{i} y \cdot\left(D_{x}+P\right)} \circ a_{B}, \quad y \in \mathbb{R}^{d} .
$$

The following lemma collects some elementary properties of $a_{B}$.

Lemma 3.3. Let $B \in \mathfrak{A}$. Then:

(1) For any Borel set $\Delta \subset \mathbb{R}^{1+d}$ :

$$
\begin{aligned}
a_{B} \mathbb{1}_{\Delta}(U) & =\left(\mathbb{1} \frac{}{\Delta+\operatorname{supp}(\widehat{B})}(U) \otimes \mathbb{1}_{\mathcal{S}^{\prime}\left(\mathbb{R}^{d}\right)}\right) \circ a_{B} \mathbb{1}_{\Delta}(U), \\
a_{B}^{*} \circ\left(\mathbb{1}_{\Delta}(U) \otimes \mathbb{1}_{\mathcal{S}\left(\mathbb{R}^{d}\right)}\right) & =\mathbb{1}_{\Delta-\operatorname{supp}(\widehat{B})}(U) a_{B}^{*} \circ\left(\mathbb{1}_{\Delta}(U) \otimes \mathbb{1}_{\mathcal{S}\left(\mathbb{R}^{d}\right)}\right) .
\end{aligned}
$$

(2) For any $f \in \mathcal{S}\left(\mathbb{R}^{d}\right)$ one has $f\left(D_{x}\right) a_{B}=a_{B_{f}}$ for

$$
B_{f}:=(2 \pi)^{-d / 2} \int \widetilde{f}(-y) B(0, y) d y=(2 \pi)^{-(1+d) / 2} \int f(-p) \widehat{B}(E, p) d E d p .
$$

Moreover, $\widehat{B_{f}}(E, p)=f(-p) \widehat{B}(E, p)$.

(3) If $\operatorname{supp}(\widehat{B})$ is compact and $f \in C^{\infty}\left(\mathbb{R}^{d}\right)$ then $f\left(D_{x}\right) a_{B}=a_{B_{f}}$.

Proof. (1) follows from (2.4), (2) is an easy consequence of (3.8) and (3) follows from (1) and (2).

The mappings $a_{B}$ have much stronger properties if $B \in \mathcal{L}_{0}$. For example, for $\Delta \Subset$ $\mathbb{R}^{1+d}$ the operator $a_{B} \mathbb{1}_{\Delta}(U)$ maps $\mathcal{H}$ into $L^{2}\left(\mathbb{R}^{d} ; \mathcal{H}\right) \simeq \mathcal{H} \otimes L^{2}\left(\mathbb{R}^{d}\right)$, as shown in Lemma 3.5 below. This is a consequence of the following important property of energy decreasing operators, proven in [Bu90].

Lemma 3.4. Let $B \in \mathfrak{A}$ be almost local and energy decreasing with $\operatorname{supp}(\widehat{B}) \Subset \mathbb{R}^{1+d}$ and $\Delta \subset \mathbb{R}^{1+d}$ be some bounded Borel set. Then there exists $c \geq 0$ such that for any $F \Subset \mathbb{R}^{d}$ one has:

$$
\left\|\int_{F}\left(B^{*} B\right)(x) \mathbb{1}_{\Delta}(U) d x\right\| \leq c .
$$

Clearly, we can let $F \nearrow \mathbb{R}^{d}$ in (3.9). In view of Lemma 3.4, it is convenient to introduce the subspace of vectors with compact energy-momentum spectrum:

$$
\mathcal{H}_{\mathrm{c}}(U):=\left\{\Psi \in \mathcal{H}: \Psi=\mathbb{1}_{\Delta}(U) \Psi, \Delta \Subset \mathbb{R}^{1+d}\right\} .
$$

There holds the following simple fact:

Lemma 3.5. Assume that $\Delta \Subset \mathbb{R}^{1+d}$ and let $B \in \mathcal{L}_{0}$. Then

$$
a_{B} \mathbb{1}_{\Delta}(U): \mathcal{H} \rightarrow \mathcal{H} \otimes L^{2}\left(\mathbb{R}^{d}\right) \text { is bounded } .
$$


Proof. We note that

$$
\mathbb{1}_{\Delta}(U) a_{B}^{*} \circ a_{B} \mathbb{1}_{\Delta}(U)=\int_{\mathbb{R}^{d}} \mathbb{1}_{\Delta}(U)\left(B^{*} B\right)(x) \mathbb{1}_{\Delta}(U) d x,
$$

and apply Lemma 3.4.

Remark 3.6. Considering $a_{B}$ as a linear operator from $\mathcal{H}$ to $\mathcal{H} \otimes L^{2}\left(\mathbb{R}^{d}\right)$ with domain $\mathcal{H}_{\mathrm{c}}(U)$, we see that $\mathcal{H} \otimes \mathcal{S}\left(\mathbb{R}^{d}\right) \subset$ Dom $a_{B}^{*}$, hence $a_{B}$ is closable.

3.4. Particle detectors. In this subsection we establish connection between the maps $a_{B}$ and the particle detectors $C_{t}$ introduced in (2.8).

Definition 3.7. Let $B \in \mathcal{L}_{0}$. For $h \in B\left(L^{2}\left(\mathbb{R}^{d}\right)\right)$ we set:

$$
N_{B}(h):=a_{B}^{*} \circ\left(\mathbb{1}_{\mathcal{H}} \otimes h\right) \circ a_{B}, \operatorname{Dom} N_{B}(h)=\mathcal{H}_{\mathrm{c}}(U) .
$$

From Lemmas 3.3 and 3.5 we obtain the following facts:

Lemma 3.8. We have:

(1) $\left\|N_{B}(h) \mathbb{1}_{\Delta}(U)\right\|_{B(\mathcal{H})} \leq c_{\Delta, B}\|h\|_{B\left(L^{2}\left(\mathbb{R}^{d}\right)\right)}$,

(2) $\forall \Delta \Subset \mathbb{R}^{1+d}, N_{B}(h) \mathbb{1}_{\Delta}(U)=\mathbb{1}_{\Delta_{1}}(U) N_{B}(h) \mathbb{1}_{\Delta}(U)$, for some $\Delta_{1} \Subset \mathbb{R}^{1+d}$.

Denoting by $h(x, y)$ the distributional kernel of $h$ we have the following expression for $N_{B}(h)$,

$$
N_{B}(h)=\int B^{*}(x) h(x, y) B(y) d x d y,
$$

which is meaningful as a quadratic form identity on $\mathcal{H}_{\mathrm{c}}(U)$. This shows that for $h \in$ $\mathcal{S}\left(T^{*} \mathbb{R}^{d}\right), h_{t}(x, \xi):=h\left(\frac{x}{t}, \xi\right), B_{t}:=B(t, 0)$ and $C_{t}$ as defined in (2.8), one has:

$$
C_{t}=N_{B_{t}}\left(h_{t}^{\mathrm{w}}\right) \text {. }
$$

3.5. Auxiliarymaps $\mathbf{a}_{\underline{B}}$. We recall that $\underline{B}=\left(B_{1}, \ldots, B_{n}\right), B_{i} \in \mathcal{L}_{0}$ and $\underline{x}=\left(x_{1}, \ldots, x_{n}\right)$, $x_{i} \in \mathbb{R}^{d}$. The Lebesgue measure in $\mathbb{R}^{n d}$ is denoted $d \underline{x}$. We state the following definition which is meaningful due to Lemma 3.5:

Definition 3.9. For $B_{1}, \ldots, B_{n} \in \mathcal{L}_{0}$ we define the linear operator:

$$
\begin{array}{ll}
a_{\underline{B}}: & \mathcal{H}_{\mathrm{c}}(U) \rightarrow \mathcal{H} \otimes L^{2}\left(\mathbb{R}^{n d}, d \underline{x}\right), \\
& \Psi \mapsto a_{\underline{B}} \Psi=\left(a_{B_{1}} \otimes \mathbb{1}_{L^{2}\left(\mathbb{R}^{(n-1) d}\right)}\right) \circ \cdots \circ\left(a_{B_{n-1}} \otimes \mathbb{1}_{L^{2}\left(\mathbb{R}^{d}\right)}\right) \circ a_{B_{n}} \Psi .
\end{array}
$$

Formally we have

$$
a_{\underline{B}} \Psi\left(x_{1}, \ldots, x_{n}\right)=B_{1}\left(x_{1}\right) \ldots B_{n}\left(x_{n}\right) \Psi .
$$

We state the following lemma, which is a direct consequence of Lemmas 3.3 and 3.5.

Lemma 3.10. Let $\Delta \Subset \mathbb{R}^{1+d}$ and let $B_{1}, \ldots, B_{n} \in \mathcal{L}_{0}$. Then:

(1) $a_{\underline{B}} \mathbb{1}_{\Delta}(U): \mathcal{H} \rightarrow \mathcal{H} \otimes L^{2}\left(\mathbb{R}^{n d}, d x\right)$ is bounded,

(2) For any $\Delta \Subset \mathbb{R}^{1+d}$ one has:

$$
\begin{gathered}
a_{\underline{B}} \mathbb{1}_{\Delta}(U)=\left(\mathbb{1}_{\Delta+\operatorname{supp}\left(\widehat{B}_{1}\right)+\cdots+\operatorname{supp}\left(\widehat{B}_{n}\right)}(U) \otimes \mathbb{1}_{L^{2}\left(\mathbb{R}^{n d}\right)}\right) \circ a_{\underline{B}} \mathbb{1}_{\Delta}(U), \\
a_{\underline{B}}^{*} \circ\left(\mathbb{1}_{\Delta}(U) \otimes \mathbb{1}_{L^{2}\left(\mathbb{R}^{n d}\right)}\right)=\mathbb{1}_{\Delta-\left(\operatorname{supp}\left(\widehat{B}_{1}\right)+\cdots+\operatorname{supp}\left(\widehat{B}_{n}\right)\right)}(U) a_{\underline{B}}^{*} \circ\left(\mathbb{1}_{\Delta}(U) \otimes \mathbb{1}_{L^{2}\left(\mathbb{R}^{n d}\right)}\right) .
\end{gathered}
$$


3.6. Some consequences of almost locality. We collect some commutator estimates which make essential use of almost locality. The proofs of Proposition 3.11 and Lemma 3.14 are given in Appendix B.

It is convenient to introduce the following functions for $N>d$ :

$$
g_{N}(\xi):=\int \mathrm{e}^{-\mathrm{i} \xi \cdot x}\langle x\rangle^{-N} d x
$$

Clearly

$$
\partial_{\xi}^{\alpha} g_{N}(\xi) \in O\left(\langle\xi\rangle^{-p}\right), \quad \forall p \in \mathbb{N},|\alpha|<N-|d|,
$$

and the operator on $L^{2}\left(\mathbb{R}^{d}\right)$ with kernel $\langle x-y\rangle^{-N}$ equals $g_{N}\left(D_{x}\right)$.

Proposition 3.11. Let $h_{i} \in B\left(L^{2}\left(\mathbb{R}^{d}\right)\right), B_{i} \in \mathcal{L}_{0}, 1 \leq i \leq n$. We set $\underline{B}=\left(B_{1}, \ldots, B_{n}\right)$, $\bar{B}=\left(B_{n}, \ldots, B_{1}\right)$ and

$$
R_{n}:=a_{\bar{B}}^{*} \circ\left(\mathbb{1}_{\mathcal{H}} \otimes h_{1} \otimes \cdots \otimes h_{n}\right) \circ a_{\underline{B}}-\prod_{i=1}^{n} N_{B_{i}}\left(h_{i}\right) .
$$

Let us fix measurable functions $\chi_{i}: \mathbb{R}^{d} \rightarrow \mathbb{R}$ with $0 \leq \chi_{i} \leq 1$ and still denote by $\chi_{i} \in B\left(L^{2}\left(\mathbb{R}^{d}\right)\right)$ the operator of multiplication by $\chi_{i}(x)$.

Then for any $\Delta \Subset \mathbb{R}^{1+d}, N \in \mathbb{N}$ there exists a constant $C_{N}\left(\Delta, B_{1}, \ldots, B_{n}\right)$ such that:

$$
\begin{aligned}
& \left\|R_{n} \mathbb{1}_{\Delta}(U)\right\|_{B(\mathcal{H})} \leq C_{N}\left(\Delta, B_{1}, \ldots, B_{n}\right) \\
& \quad \times\left(\sum_{i=1}^{n}\left(\left\|h_{i}\left(1-\chi_{i}\right)\right\|_{B\left(L^{2}\left(\mathbb{R}^{d}\right)\right)}+\left\|\left(1-\chi_{i}\right) h_{i}\right\|_{B\left(L^{2}\left(\mathbb{R}^{d}\right)\right)}\right) \prod_{j \neq i}\left\|h_{j}\right\|_{B\left(L^{2}\left(\mathbb{R}^{d}\right)\right)}\right. \\
& \left.\quad+\sum_{i \neq j}\left\|\chi_{i} g_{N}\left(D_{x}\right) \chi_{j}\right\|_{B\left(L^{2}\left(\mathbb{R}^{d}\right)\right)} \prod_{i=1}^{n}\left\|h_{i}\right\|_{B\left(L^{2}\left(\mathbb{R}^{d}\right)\right)}\right) .
\end{aligned}
$$

Remark 3.12. Let us explain the meaning of Proposition 3.11. By almost locality we expect $R_{n}$ to be small if the operators $h_{i}$ are 'localized' in distant regions of configuration space. This is easy to prove if $h_{i}=h_{i}(x)$ for functions $h_{i}$ with compact, pairwise disjoint supports. In the general case we pick functions $\chi_{i}$ such that the operators $h_{i}\left(1-\chi_{i}\right)$ and $\left(1-\chi_{i}\right) h_{i}$ are small, i.e., $h_{i}$ is 'localized' in the support of $\chi_{i}$. If these supports are far away from each other, then the operators $\chi_{i} g_{N}\left(D_{x}\right) \chi_{j}$, and hence $R_{n}$, will also be small.

Corollary 3.13. Let $\underline{B}$ be as in Proposition 3.11 and $\tilde{h}_{i} \in C_{0}^{\infty}\left(T^{*} \mathbb{R}^{d}\right)$ with $\pi_{x} \operatorname{supp} \tilde{h}_{i} \cap$ $\pi_{x} \operatorname{supp} \tilde{h}_{j}=\emptyset$ for $i \neq j$, where $\pi_{x}: T^{*} \mathbb{R}^{d} \rightarrow \mathbb{R}^{d}$ is the projection on the configuration space. Let $R_{n}(t)$ be as in (3.13) with the operators $h_{i}$ replaced with $\tilde{h}_{i, t}^{\mathrm{w}}$. Then for any $\Delta \Subset \mathbb{R}^{1+d}$ one has:

$$
\left\|R_{n}(t) \mathbb{1}_{\Delta}(U)\right\|_{B(\mathcal{H})} \in O\left(t^{-\infty}\right) .
$$


Proof. We choose functions $\tilde{\chi}_{i} \in C_{0}^{\infty}\left(\mathbb{R}^{d}\right)$ such that $0 \leq \tilde{\chi}_{i} \leq 1, \tilde{\chi}_{i}(x)=1$ near $\pi_{x} \operatorname{supp} \tilde{h}_{i}$ and $\operatorname{supp} \tilde{\chi}_{i}$ pairwise disjoint. We set $\tilde{\chi}_{i, t}(x):=\tilde{\chi}_{i}(x / t)$ and denote the corresponding operators on $B\left(L^{2}\left(\mathbb{R}^{d}\right)\right)$ by the same symbol. We apply Proposition 3.11 to $h_{i}=\tilde{h}_{i, t}^{\mathrm{w}}$ and $\chi_{i}=\tilde{\chi}_{i, t}$. By Proposition $3.1(6),\left\|\tilde{h}_{i, t}^{\mathrm{w}}\left(1-\tilde{\chi}_{i, t}\right)\right\|+\left\|\left(1-\tilde{\chi}_{i, t}\right) \tilde{h}_{i, t}^{\mathrm{w}}\right\| \in$ $O\left(t^{-\infty}\right)$. Similarly we can estimate the operator norm of $\tilde{\chi}_{i, t} g_{N}\left(D_{x}\right) \tilde{\chi}_{j, t}$ by its HilbertSchmidt norm which equals

$$
\left(\int \tilde{\chi}_{i, t}^{2}(x)\langle x-y\rangle^{-2 N} \tilde{\chi}_{j, t}^{2}(y) d x d y\right)^{\frac{1}{2}} \in O\left(t^{d-N}\right) .
$$

Since $N$ is arbitrary we obtain the lemma.

The following lemma is similar to Proposition 3.11. Its proof is given in Appendix B.2.

Lemma 3.14. Let $B_{1}, B_{2} \in \mathcal{L}_{0}, h_{1} \in B\left(L^{2}\left(\mathbb{R}^{d}\right)\right)$ and $g_{2} \in L^{2}\left(\mathbb{R}^{d}\right)$. Let us fix measurable functions $\chi_{i}: \mathbb{R}^{d} \rightarrow \mathbb{R}, i=1,2$, with $0 \leq \chi_{i} \leq 1$ and still denote by $\chi_{i} \in B\left(L^{2}\left(\mathbb{R}^{d}\right)\right)$ the operator of multiplication by $\chi_{i}$. Then for any $\Delta \Subset \mathbb{R}^{1+d}, N \in \mathbb{N}$ there exists $C_{N}\left(\Delta, B_{1}, B_{2}\right)$ such that:

$$
\begin{aligned}
& \left\|\left[N_{B_{1}}\left(h_{1}\right), B_{2}^{*}\left(g_{2}\right)\right] \mathbb{1}_{\Delta}(U)\right\|_{B(\mathcal{H})} \leq C_{N}\left(\Delta, B_{1}, B_{2}\right) \\
& \quad \times\left(\left\|h_{1}\left(1-\chi_{1}\right)\right\|_{B\left(L^{2}\left(\mathbb{R}^{d}\right)\right)}\left\|g_{2}\right\|_{L^{2}\left(\mathbb{R}^{d}\right)}+\left\|\left(1-\chi_{1}\right) h_{1}\right\|_{B\left(L^{2}\left(\mathbb{R}^{d}\right)\right)}\left\|g_{2}\right\|_{L^{2}\left(\mathbb{R}^{d}\right)}\right. \\
& \quad+\left\|h_{1}\right\|_{B\left(L^{2}\left(\mathbb{R}^{d}\right)\right)}\left\|\left(1-\chi_{2}\right) g_{2}\right\|_{L^{2}\left(\mathbb{R}^{d}\right)}+\left\|h_{1}\right\|_{B\left(L^{2}\left(\mathbb{R}^{d}\right)\right)}\left\|g_{2}\right\|_{L^{2}\left(\mathbb{R}^{d}\right)} \\
& \left.\quad \times\left\|\chi_{1} g_{N}\left(D_{x}\right) \chi_{2}\right\|_{B\left(L^{2}\left(\mathbb{R}^{d}\right)\right)}\right) .
\end{aligned}
$$

\section{An Intermediate Convergence Argument}

Theorem 4.1 and Lemma 4.2 below essentially reduce the proof of Theorem 2.8 to an argument adapted from non-relativistic scattering theory, which will be presented in Sect. 5. The results of the present section generalize to arbitrary $n$ the corresponding arguments from [DG12], where we studied the case of $n=2$ detectors. The discussion in Sect. 5 will be very different from [DG12], however.

Let $B_{i} \in \mathcal{L}_{0}, h_{i} \in \mathcal{S}\left(T^{*} \mathbb{R}^{d}\right), i=1, \ldots, n$, and set

$$
h_{i, t}(x, \xi):=h_{i}\left(\frac{x}{t}, \xi\right), \quad N_{B_{i}}\left(h_{i}^{\mathrm{w}}, t\right):=N_{B_{i, t}}\left(h_{i, t}^{\mathrm{w}}\right) .
$$

We recall the notation $\underline{x}=\left(x_{1}, \ldots, x_{n}\right)$ and $\tilde{\omega}\left(D_{\underline{x}}\right)=\omega\left(D_{x_{1}}\right)+\cdots+\omega\left(D_{x_{n}}\right)$, where $\tilde{\omega}\left(D_{\underline{x}}\right)$ is an operator acting on $L^{2}\left(\mathbb{R}^{n d}\right)$.

Theorem 4.1. Let $\Delta \subset \mathbb{R}^{1+d}$ be an open bounded set, $B_{1}, \ldots, B_{n} \in \mathcal{L}_{0}$ be s.t. $\underline{B}$ is $\Delta$-admissible and let $h_{1}, \ldots, h_{n} \in \mathcal{S}\left(T^{*} \mathbb{R}^{d}\right)$ be s.t. $\pi_{x}\left(\operatorname{supp} h_{i}\right)$ are pairwise disjoint. Let $\mathrm{H}(\underline{x}, \underline{\xi}):=\prod_{i=1}^{n} h_{i}\left(x_{i}, \xi_{i}\right)$ and $\mathrm{H}_{t}(\underline{x}, \underline{\xi}):=\mathrm{H}(\underline{x} / t, \underline{\xi})$.

We setfor $\Psi \in \mathbb{1}_{\Delta}(U) \mathcal{H}$ :

$$
F_{t}:=\left(\langle\Omega| \otimes \mathbb{1}_{L^{2}\left(\mathbb{R}^{n d}\right)}\right) \circ a_{\underline{B}} \mathrm{e}^{-i t H} \Psi \in L^{2}\left(\mathbb{R}^{n d}\right),
$$

so that

$$
F_{t}\left(x_{1}, \ldots, x_{n}\right)=\left(\Omega \mid B_{1}\left(t, x_{1}\right) \ldots B_{n}\left(t, x_{n}\right) \Psi\right)_{\mathcal{H}}, \quad\left(x_{1}, \ldots, x_{n}\right) \in \mathbb{R}^{n d} .
$$


Assume that:

$$
F_{+}:=\lim _{t \rightarrow \infty} \mathrm{e}^{\mathrm{i} t \tilde{\omega}\left(D_{\underline{x}}\right)} \mathrm{H}_{t}^{\mathrm{W}} F_{t} \text { exists. }
$$

Then

$$
\lim _{t \rightarrow \infty} N_{B_{1}}\left(h_{1}^{\mathrm{w}}, t\right) \ldots N_{B_{n}}\left(h_{n}^{\mathrm{w}}, t\right) \Psi
$$

exists and belongs to $\mathbb{1}_{\Delta}(U) \mathcal{H}_{n}^{+}$.

Proof. Applying Corollary 3.13, we get:

$$
N_{B_{1}}\left(h_{1, t}^{\mathrm{w}}\right) \ldots N_{B_{n}}\left(h_{n, t}^{\mathrm{w}}\right) \mathbb{1}_{\Delta}(U)=a_{\bar{B}}^{*} \circ\left(\mathbb{1}_{\mathcal{H}} \otimes \mathrm{H}_{t}^{\mathrm{w}}\right) \circ a_{\underline{B}} \mathbb{1}_{\Delta}(U)+O\left(t^{-\infty}\right) .
$$

By the $\Delta$-admissibility of $\underline{B}$ (more precisely, property (2.13)) and Lemma 3.10 we have:

$$
a_{\underline{B}} \mathbb{1}_{\Delta}(U)=\left(\mathbb{1}_{\{0\}}(U) \otimes \mathbb{1}_{L^{2}\left(\mathbb{R}^{n d}\right)}\right) \circ a_{\underline{B}} \mathbb{1}_{\Delta}(U)=\left(|\Omega\rangle\langle\Omega| \otimes \mathbb{1}_{L^{2}\left(\mathbb{R}^{n d}\right)}\right) \circ a_{\underline{B}} \mathbb{1}_{\Delta}(U),
$$

using the spectrum condition (2.2). Therefore we have:

$$
\begin{aligned}
\mathrm{e}^{\mathrm{i} t H} N_{B_{1}}\left(h_{1, t}^{\mathrm{w}}\right) \ldots N_{B_{n}}\left(h_{n, t}^{\mathrm{w}}\right) \mathrm{e}^{-\mathrm{i} t H} \Psi & =\mathrm{e}^{\mathrm{i} t H} a_{\bar{B}}^{*}\left(\Omega \otimes \mathrm{H}_{t}^{\mathrm{w}} F_{t}\right)+O\left(t^{-\infty}\right) \\
& =\mathrm{e}^{\mathrm{i} t H} a_{B}^{*}\left(\Omega \otimes \mathrm{e}^{-\mathrm{i} t \tilde{\omega}\left(D_{\underline{x}}\right)} F_{+}\right)+o\left(t^{0}\right),
\end{aligned}
$$

by (4.3). We set

$$
S_{t}: L^{2}\left(\mathbb{R}^{n d}\right) \ni F \mapsto \mathrm{e}^{\mathrm{i} t H} a_{\bar{B}}^{*}\left(\Omega \otimes \mathrm{e}^{-\mathrm{i} t \tilde{\omega}\left(D_{\underline{x}}\right)} F\right) \in \mathcal{H} .
$$

By Lemma 3.10 the family $S_{t}$ is uniformly bounded in norm. Moreover if $g_{1}, \ldots, g_{n}$ are positive energy $\mathrm{KG}$ solutions with disjoint velocity supports (defined in Sect. 6.1) and $f_{1}, \ldots, f_{n} \in \mathcal{S}\left(\mathbb{R}^{d}\right)$ are their initial data, then

$$
S_{t}\left(f_{1} \otimes \cdots \otimes f_{n}\right)=B_{1, t}^{*}\left(g_{1, t}\right) \ldots B_{n, t}^{*}\left(g_{n, t}\right) \Omega,
$$

where the Haag-Ruelle creation operators $B_{i, t}^{*}\left(g_{i, t}\right)$ are defined in Sect. 6.2. From Theorem 6.5 we know that $\lim _{t \rightarrow \infty} S_{t}\left(f_{1} \otimes \cdots \otimes f_{n}\right)$ exists. By linearity and density arguments, using the uniform boundedness of $S_{t}$, we conclude that $\lim _{t \rightarrow \infty} S_{t} F$ exists for any $F \in L^{2}\left(\mathbb{R}^{n d}\right)$. In view of (4.5) this implies the existence of the limit in (4.4). It is also clear from the approximation argument above that this limit belongs to $\mathcal{H}_{n}^{+}$. Due to $\Delta$-admissibility of $\underline{B}$ it belongs to the range of $\mathbb{1}_{\Delta}(U)$.

The proof of the existence of the limit (4.3) will be given in the next section. The key input is the fact that $F_{t}$ solves a Schrödinger equation with Hamiltonian $\tilde{\omega}\left(D_{x}\right)$ and a source term $R_{t}$ whose $L^{2}$ norm decreases very fast when $t \rightarrow+\infty$ outside of the collision planes $\left\{\underline{x} \in \mathbb{R}^{n d}: x_{i}=x_{j}\right\}, i \neq j$. This is the content of the following lemma:

Lemma 4.2. Let $F_{t}$ be defined in (4.2). Then:

(1) $F_{t}$ is uniformly bounded in $L^{2}\left(\mathbb{R}^{n d}\right)$,

(2) $t \mapsto F_{t} \in L^{2}\left(\mathbb{R}^{n d}\right)$ is $C^{1}$ with

$$
\partial_{t} F_{t}=-\mathrm{i} \tilde{\omega}\left(D_{\underline{x}}\right) F_{t}+R_{t},
$$

where $R_{t}$ satisfies the assumptions of Lemma 5.1 below. 
Proof. We have $F_{t}\left(x_{1}, \ldots, x_{n}\right)=\left(\Omega \mid B_{1}\left(t, x_{1}\right) \ldots B_{n}\left(t, x_{n}\right) \Psi\right)_{\mathcal{H}}$, which is uniformly bounded in $L^{2}\left(\mathbb{R}^{n d}\right)$ by Lemma 3.10. We set $\dot{B}_{i}:=\partial_{s} B_{i}(s, 0)_{\mid s=0}$ and note that since $\Psi \in \mathcal{H}_{\mathrm{c}}(U)$, the map $t \mapsto F_{t} \in L^{2}\left(\mathbb{R}^{n d}\right)$ is $C^{1}$ with:

$$
\begin{aligned}
\partial_{t} F_{t}(\underline{x}) & =\sum_{i=1}^{n}\left(\Omega \mid B_{1}\left(t, x_{1}\right) \ldots \dot{B}_{i}\left(t, x_{i}\right) \ldots B_{n}\left(t, x_{n}\right) \Psi\right)_{\mathcal{H}} \\
& =\sum_{i=1}^{n}\left(\Omega \mid \dot{B}_{i}\left(t, x_{i}\right) B_{1}\left(t, x_{1}\right) \ldots \check{i} \ldots B_{n}\left(t, x_{n}\right) \Psi\right)_{\mathcal{H}}+R_{1, t}(\underline{x}),
\end{aligned}
$$

where

$$
\begin{aligned}
R_{1, t}(\underline{x})= & \sum_{i=2}^{n} \sum_{j=1}^{i-1}\left(\Omega \mid B_{1}\left(t, x_{1}\right) \ldots B_{j-1}\left(t, x_{j-1}\right)\left[B_{j}\left(t, x_{j}\right), \dot{B}_{i}\left(t, x_{i}\right)\right]\right. \\
& \left.\times B_{j+1}\left(t, x_{j+1}\right) \ldots \check{i} \ldots B_{n}\left(t, x_{n}\right) \Psi\right)_{\mathcal{H}} .
\end{aligned}
$$

(For $j=1$ in the above sum we set $B_{1}\left(t, x_{1}\right) \ldots B_{j-1}\left(t, x_{j-1}\right):=I$ and for $j=$ $i-1$ we set $B_{j+1}\left(t, x_{j+1}\right) \ldots \check{i} \ldots B_{n}\left(t, x_{n}\right)=B_{i+1}\left(t, x_{i+1}\right) \ldots B_{n}\left(t, x_{n}\right)$ which is to be understood as $I$ if $i=n)$. Note that $\dot{B}_{i}$ are again almost local by definition of $\mathcal{L}_{0}$. Using almost locality of $B_{i}, \dot{B}_{i}$, we easily obtain that $R_{1, t}$ satisfies the assumptions of Lemma 5.1 below.

There holds for any $\Phi \in \mathcal{H}$ :

$$
\begin{aligned}
\left(\Omega \mid B_{i}\left(t, x_{i}\right) \Phi\right)_{\mathcal{H}} & =\left(\Omega \mid \mathbb{1}_{\{0\}}(U) B_{i}\left(t, x_{i}\right) \Phi\right)_{\mathcal{H}}=\left(\Omega \mid B_{i}\left(t, x_{i}\right) \mathbb{1}_{H_{m}}(U) \Phi\right)_{\mathcal{H}} \\
& =\left(\Omega \mid B_{i}\left(x_{i}\right) \mathrm{e}^{-\mathrm{i} t \omega(P)} \Phi\right)_{\mathcal{H}}=\mathrm{e}^{-\mathrm{i} t \omega\left(D_{x_{i}}\right)}\left(\Omega \mid B_{i}\left(x_{i}\right) \Phi\right),
\end{aligned}
$$

using (2.4), (2.11) and finally (3.8). Differentiating this identity we obtain

$$
\left(\Omega \mid \dot{B}_{i}\left(t, x_{i}\right) \Phi\right)_{\mathcal{H}}=-\mathrm{i} \omega\left(D_{x_{i}}\right)\left(\Omega \mid B_{i}\left(t, x_{i}\right) \Phi\right)_{\mathcal{H}}
$$

We get from (4.6) and (4.7) that

$$
\begin{aligned}
\partial_{t} F_{t}(\underline{x}) & =-\sum_{i=1}^{n} \mathrm{i} \omega\left(D_{x_{i}}\right)\left(\Omega \mid B_{i}\left(t, x_{i}\right) B_{1}\left(t, x_{1}\right) \ldots \check{i} \ldots B_{n}\left(t, x_{n}\right) \Psi\right)_{\mathcal{H}}+R_{1, t}(\underline{x}) \\
& =-\mathrm{i} \tilde{\omega}\left(D_{\underline{x}}\right) F_{t}\left(x_{1}, \ldots, x_{n}\right)+R_{1, t}(\underline{x})+R_{2, t}(\underline{x}),
\end{aligned}
$$

where

$$
\begin{aligned}
R_{2, t}(\underline{x}):= & -\sum_{i=2}^{n} \sum_{j=1}^{i-1} \mathrm{i} \omega\left(D_{x_{i}}\right)\left(\Omega \mid B_{1}\left(t, x_{1}\right) \ldots B_{j-1}\left(t, x_{j-1}\right)\left[B_{i}\left(t, x_{i}\right), B_{j}\left(t, x_{j}\right)\right]\right. \\
& \left.\times B_{j+1}\left(t, x_{j+1}\right) \ldots \breve{i} \ldots B_{n}\left(t, x_{n}\right) \Psi\right)_{\mathcal{H}} .
\end{aligned}
$$

To conclude the proof it suffices to show that $R_{2, t}$ satisfies the assumptions of Lemma 5.1. To this end, we note that for any $\Phi_{1}, \Phi_{2} \in \mathcal{H}_{\mathrm{c}}(U)$ we can write

$$
\omega\left(D_{x_{i}}\right)\left(\Phi_{1} \mid\left[B_{i}\left(t, x_{i}\right), B_{j}\left(t, x_{j}\right)\right] \Phi_{2}\right)=\left(\Phi_{1} \mid\left[C_{i}\left(t, x_{i}\right), B_{j}\left(t, x_{j}\right)\right] \Phi_{2}\right),
$$

where, by Lemma 3.3 (3),

$$
C_{i}=(2 \pi)^{-d / 2} \int f(x) B_{i}(0, x) d x, f \in \mathcal{S}\left(\mathbb{R}^{d}\right), \widehat{f}(-p) \equiv \omega(p) \text { near } \operatorname{supp}\left(\widehat{B}_{i}\right) .
$$

Since $C_{i}$ are almost local, we can argue as in the case of $R_{1, t}$ and the proof is complete. 


\section{Scattering for Schrödinger Equations with Source Terms}

In this section we give the proof of the existence of the limit

$$
F_{+}=\lim _{t \rightarrow+\infty} \mathrm{e}^{\mathrm{i} t \tilde{\omega}\left(D_{\underline{x}}\right)} \mathrm{H}_{t}^{\mathrm{w}} F_{t},
$$

appearing in Theorem 4.1. The proof relies on the fact that $F_{t}$ satisfies a Schrödinger equation with a source term $R_{t}$ as shown in Lemma 4.2 above. To control the influence of $R_{t}$, we need the following fact.

Lemma 5.1. Let $R_{t} \in L^{2}\left(\mathbb{R}^{\text {nd }}\right)$ satisfy

(i) $\left\|R_{t}\right\|_{L^{2}\left(\mathbb{R}^{n d}\right)} \in O\left(t^{N_{0}}\right), \quad N_{0} \in \mathbb{N}$,

(ii) $\left|R_{t}(\underline{x})\right| \leq C_{N} \sum_{i \neq j}\left\langle x_{i}-x_{j}\right\rangle^{-N}, \quad \forall N \in \mathbb{N}$, uniformly in $t$.

Let $\mathrm{H} \in \mathcal{S}\left(T^{*} \mathbb{R}^{n d}\right)$ be such that

$$
\pi_{\underline{x}}(\operatorname{supp~} \mathrm{H}) \cap D_{0}=\emptyset,
$$

where $D_{0} \subset \mathbb{R}^{\text {nd }}$ is defined in (2.14). Then

$$
\left\|\mathrm{H}_{t}^{\mathrm{w}} R_{t}\right\|_{L^{2}\left(\mathbb{R}^{n d}\right)}=O\left(t^{-\infty}\right) .
$$

Remark 5.2. Note that symbols which are admissible (in the sense of Definition 2.7) satisfy (5.2).

Proof. Let $K_{t}(\underline{x}, \underline{y})=\breve{\mathrm{H}}((\underline{x}+\underline{y}) / 2 t, \underline{x}-\underline{y})$ be the distributional kernel of $\mathrm{H}_{t}^{\mathrm{w}}$. Let $\delta>0, \chi \in C_{0}^{\infty}\left(\mathbb{R}^{n} d\right)$ with $\chi \equiv 1$ near 0 and set $K_{1, t}(\underline{x}, \underline{y})=K_{t}(\underline{x}, \underline{y}) \chi((\underline{x}-\underline{y}) / \delta t)$, $K_{2, t}=K_{t}-K_{1, t}$. Since $\check{\mathrm{H}} \in \mathcal{S}\left(\mathbb{R}^{n d} \times \mathbb{R}^{n d}\right)$ we easily see that $\left\|K_{2, t}\right\|_{L^{2}\left(\mathbb{R}^{2 n d}\right)} \in$ $O\left(t^{-\infty}\right)$. Still denoting by $K_{2, t}$ the operator with kernel $K_{2, t}$, we deduce from (i) that $\left\|K_{2, t} R_{t}\right\|_{L^{2}\left(\mathbb{R}^{n d}\right)} \in O\left(t^{-\infty}\right)$. On the other hand we have using (ii) and (5.2):

$$
\begin{aligned}
& \left\|K_{1, t} R_{t}\right\|_{L^{2}\left(\mathbb{R}^{n d}\right)}^{2} \\
& \quad \leq C_{N} t^{3 n d} \sum_{i \neq j} \int \mid \int \widetilde{\mathrm{H}}\left((\underline{x}+\underline{y}) / 2, t(\underline{x}-\underline{y})\left|\chi((\underline{x}-\underline{y}) / \delta)\left\langle t\left(y_{i}-y_{j}\right)\right\rangle^{-N} d \underline{y}\right|^{2} d \underline{x} .\right.
\end{aligned}
$$

For $\delta \ll \epsilon$ the integrand is supported in $\left\{\left|y_{i}-y_{j}\right| \geq \epsilon / 2\right\}$, hence the integral is $O\left(t^{3 n d-2 N}\right)$. This concludes the proof.

The main ingredient of the proof of existence of the limit in (5.1) is a novel propagation estimate established in Proposition 5.3 below. As a preparation we recall that $F_{t}$ is defined in (4.2) and note that for $M \in \mathcal{S}\left(T^{*} \mathbb{R}^{n d}\right)$ and $M_{t}(\underline{x}, \underline{\xi})=M(\underline{x} / t, \underline{\xi})$ we have by Proposition 3.1 (3):

$$
\begin{aligned}
\mathcal{D} M_{t}^{\mathrm{w}} & :=\partial_{t} M_{t}^{\mathrm{W}}+\left[\tilde{\omega}\left(D_{\underline{x}}\right), \mathrm{i} M_{t}^{\mathrm{w}}\right]=t^{-1}(d M)_{t}^{\mathrm{w}}+O\left(t^{-2}\right), \text { for } \\
d M(\underline{x}, \underline{\xi}) & :=\left.\left(\partial_{t} M_{t}-\left\{\tilde{\omega}(\underline{\xi}), M_{t}\right\}\right)\right|_{t=1}=-(\underline{x}-\nabla \tilde{\omega}(\underline{\xi})) \cdot \nabla_{\underline{x}} M(\underline{x}, \underline{\xi}),
\end{aligned}
$$

where $\{\cdot, \cdot\}$ is the Poisson bracket. In the remaining part of this section we set $\|\cdot\|:=$ $\|\cdot\|_{L^{2}\left(\mathbb{R}^{n d}\right)}$. 
Proposition 5.3. Let $\mathrm{H} \in C_{0}^{\infty}\left(T^{*} \mathbb{R}^{n d} ; \mathbb{R}^{n d}\right)$ be admissible in the sense of Definition 2.7. Then

$$
\begin{gathered}
\int_{1}^{+\infty}\left\|\left(\underline{x} / t-\nabla \tilde{\omega}\left(D_{\underline{x}}\right)\right) \cdot \mathrm{H}_{t}^{\mathrm{w}} F_{t}\right\|^{2} \frac{d t}{t}<\infty \\
\int_{1}^{+\infty}\left\|\left(\underline{x} / t-\nabla \tilde{\omega}\left(D_{\underline{x}}\right)\right) \cdot \mathrm{H}_{t}^{\mathrm{w}} \mathrm{e}^{-\mathrm{i} t \tilde{\omega}\left(D_{\underline{x}}\right)} u\right\|^{2} \frac{d t}{t} \leq c\|u\|^{2}, \quad u \in L^{2}\left(\mathbb{R}^{n d}\right) .
\end{gathered}
$$

Proof. Let $K$ and $\epsilon$ be as in Definition 2.7 and choose $0<\lambda \ll \epsilon$. Let $\chi \in C^{\infty}(\mathbb{R})$ with $\chi(s)=1$ for $s \leq-1, \chi(s)=0$ for $s \geq(1+\lambda)^{2}, \chi^{\prime}(s) \leq 0$ for $s \in \mathbb{R}$ and $\chi^{\prime}(s) \leq-\frac{1}{2}$ for $0 \leq s \leq 1$. Let $\chi_{1} \in C_{0}^{\infty}\left(\mathbb{R}^{n d}\right)$ be equal to 1 on $K$ and vanish outside of $K+\tilde{\mathcal{B}}(0, \lambda)$. We set

$$
M(\underline{x}, \underline{\xi}):=\chi\left(\epsilon^{-2}|\underline{x}-\nabla \tilde{\omega}(\underline{\xi})|^{2}\right) \chi_{1}(\nabla \tilde{\omega}(\underline{\xi})) .
$$

By Definition $2.7, K+\tilde{\mathcal{B}}(0, \lambda) \subset \tilde{\mathcal{B}}(0,1)$, hence $M \in \mathcal{S}\left(T^{*} \mathbb{R}^{n d}\right)$ and we obtain from (5.5)

$$
d M(\underline{x}, \underline{\xi})=-\epsilon^{-2}|\underline{x}-\nabla \tilde{\omega}(\underline{\xi})|^{2} \chi^{\prime}\left(\epsilon^{-2}|\underline{x}-\nabla \tilde{\omega}(\underline{\xi})|^{2}\right) \chi_{1}(\nabla \tilde{\omega}(\underline{\xi}))
$$

Making use of the properties of $\chi$ and $\chi_{1}$ we obtain for some $c>0$ :

$$
\begin{gathered}
d M(\underline{x}, \underline{\xi}) \geq c \mathbb{1}_{\mathcal{B}(0, \epsilon)}(\underline{x}-\nabla \tilde{\omega}(\underline{\xi})) \mathbb{1}_{K}(\nabla \tilde{\omega}(\underline{\xi}))|\underline{x}-\nabla \tilde{\omega}(\underline{\xi})|^{2}, \\
\pi_{x}(\operatorname{supp} M) \subset \mathbb{R}^{n d} \backslash D_{0} .
\end{gathered}
$$

Relation (5.8) holds for $\lambda \ll \epsilon$ and follows from the facts that for $y \in K+\tilde{\mathcal{B}}(0, \lambda)$ we have $\left|y_{i}-y_{j}\right| \geq 2(\epsilon-\lambda)$ for $i \neq j$ and $|\underline{z}| \leq \epsilon(1+\lambda)$ implies $\left|z_{i}\right|+\left|z_{j}\right| \leq \sqrt{2} \epsilon(1+\lambda)$.

Relation (5.8) and Lemma 5.1 imply that $\left\|M_{t}^{\mathrm{w}} R_{t}\right\|,\left\|\left(M_{t}^{\mathrm{W}}\right)^{*} R_{t}\right\|$ belong to $L^{1}(\mathbb{R}, d t)$. Properties (5.7), (5.4) give

$$
\mathcal{D} M_{t}^{\mathrm{w}} \geq \frac{c}{t}\left(\underline{x} / t-\nabla \tilde{\omega}\left(D_{\underline{x}}\right)\right) \cdot \mathrm{H}_{t}^{\mathrm{W} *} \mathrm{H}_{t}^{\mathrm{w}} \cdot\left(\underline{x} / t-\nabla \tilde{\omega}\left(D_{\underline{x}}\right)\right)+O\left(t^{-2}\right),
$$

using Proposition 3.1 (1), (5), (4). Applying Lemma A.1 we obtain the first statement of (5.6). The proof of the second is similar.

Theorem 5.4. Let $F_{t}$, be defined in (4.2) and $\mathrm{H} \in C_{0}^{\infty}\left(\mathbb{R}^{n d}\right)$ be admissible in the sense of Definition 2.7. Then the limit

$$
F_{+}=\lim _{t \rightarrow+\infty} \mathrm{e}^{\mathrm{i} t \tilde{\omega}\left(D_{\underline{x}}\right)} \mathrm{H}_{t}^{\mathrm{w}} F_{t} \text { exists. }
$$

Proof. We proceed similarly as in the proof of [DG97, Prop. 4.4.5]. We apply (5.5) to

$$
M(\underline{x}, \underline{\xi}):=\mathrm{H}(\underline{x}, \underline{\xi})-(\underline{x}-\nabla \tilde{\omega}(\underline{\xi})) \cdot\left(\nabla_{\underline{x}} \mathrm{H}\right)(\underline{x}, \underline{\xi}),
$$

which yields

$$
d M(\underline{x}, \underline{\xi})=(\underline{x}-\nabla \tilde{\omega}(\underline{\xi})) \cdot \nabla_{\underline{x}}^{2} \mathrm{H}(\underline{x}, \underline{\xi}) \cdot(\underline{x}-\nabla \tilde{\omega}(\underline{\xi})) .
$$


By the admissibility of $\mathrm{H}$, we can find $\mathrm{H}_{1} \in C_{0}^{\infty}\left(T^{*} \mathbb{R}^{n d} ; \mathbb{R}^{n d}\right)$ which is also admissible s.t.

$$
d M(\underline{x}, \underline{\xi})=(\underline{x}-\nabla \tilde{\omega}(\underline{\xi})) \cdot \mathrm{H}_{1}(\underline{x}, \underline{\xi}) \nabla_{\underline{x}}^{2} \mathrm{H}(\underline{x}, \underline{\xi}) \mathrm{H}_{1}(\underline{x}, \underline{\xi}) \cdot(\underline{x}-\nabla \tilde{\omega}(\underline{\xi})) \cdot
$$

By pseudodifferential calculus (see Proposition 3.1 (4), (5)) and (5.4) one has for $u \in$ $L^{2}\left(\mathbb{R}^{n d}\right)$ :

$$
\begin{aligned}
\left|\left(u \mid \mathcal{D} M_{t}^{\mathrm{W}} F_{t}\right)\right| \leq & \frac{c}{t}\left\|\left(\underline{x} / t-\nabla \tilde{\omega}\left(D_{\underline{x}}\right)\right) \cdot \mathrm{H}_{1, t}^{\mathrm{W}} u\right\|\left\|\left(\underline{x} / t-\nabla \tilde{\omega}\left(D_{\underline{x}}\right)\right) \cdot \mathrm{H}_{1, t}^{\mathrm{W}} F_{t}\right\| \\
& +O\left(t^{-2}\right)\|u\| .
\end{aligned}
$$

Since $M$ satisfies the assumptions imposed on $\mathrm{H}$ in Lemma 5.1, we obtain

$$
\left\|M_{t}^{\mathrm{w}} R_{t}\right\|,\left\|\left(M_{t}^{\mathrm{w}}\right)^{*} R_{t}\right\| \in O\left(t^{-\infty}\right) .
$$

Making use of (5.12), (5.13), Proposition 5.3 and Lemma A.3, we get that

$$
\lim _{t \rightarrow+\infty} \mathrm{e}^{\mathrm{i} t \tilde{\omega}\left(D_{\underline{x}}\right)} M_{t} F_{t} \text { exists. }
$$

To conclude the proof, it suffices to verify that $M_{t}-\mathrm{H}_{t}$ does not contribute to the above limit. For admissible $\mathrm{H}_{2} \in C_{0}^{\infty}\left(T^{*} \mathbb{R}^{n d} ; \mathbb{R}^{n d}\right)$, we set

$$
N(\underline{x}, \underline{\xi}):=(\underline{x}-\nabla \tilde{\omega}(\underline{\xi})) \cdot \mathrm{H}_{2}(\underline{x}, \underline{\xi}) .
$$

We have to show that

$$
\lim _{t \rightarrow \infty}\left\|N_{t}^{\mathrm{w}} F_{t}\right\|=0
$$

By Proposition 5.3 the limit must be zero if it exists. To prove the existence of the limit, we first note that by pseudodifferential calculus

$$
\left(N_{t}^{\mathrm{W}}\right)^{*} N_{t}^{\mathrm{w}}=\left(|N|_{t}^{2}\right)^{\mathrm{w}}+O\left(t^{-1}\right) .
$$

Next, making use of relation (5.5), we obtain

$$
\begin{aligned}
d|N|^{2}(\underline{x}, \underline{\xi})= & (\underline{x}-\nabla \tilde{\omega}(\underline{\xi})) \cdot N_{1}(\underline{x}, \underline{\xi}) \cdot(\underline{x}-\nabla \tilde{\omega}(\underline{\xi})), \text { for } \\
N_{1, i, j}(\underline{x}, \underline{\xi}):= & -(\underline{x}-\nabla \tilde{\omega}(\underline{\xi})) \cdot \nabla_{\underline{x}}\left(\mathrm{H}_{2, i}^{*} \mathrm{H}_{2, j}\right)(\underline{x}, \underline{\xi})-2\left(\mathrm{H}_{2, i}^{*} \mathrm{H}_{2, j}\right)(\underline{x}, \underline{\xi}), \\
& i, j=1, \ldots, n d .
\end{aligned}
$$

Since $|N|^{2}$ is admissible, we obtain by Lemma 5.1:

$$
\left\|\left(|N|_{t}^{2}\right)^{\mathrm{w}} R_{t}\right\| \in O\left(t^{-\infty}\right) .
$$

Exploiting the admissibility of $N_{1}$, we can rewrite (5.15) as in (5.10), (5.11) above and conclude the existence of the limit (5.14) from (5.4), Proposition 5.3 and Lemma A.2.

\section{Haag-Ruelle Scattering Theory}

In this section we collect some basic facts concerning the Haag-Ruelle scattering theory, which we need for the proof of Theorem 2.9. For the reader's convenience we give a self-contained presentation of this classical topic in the setting of the present paper. In the special case of two-body scattering we presented a similar discussion in [DG12]. 


\subsection{Positive energy solutions of the Klein-Gordon equation.}

Definition 6.1. Let $f \in \mathcal{S}\left(\mathbb{R}^{d}\right)$ be such that $\widehat{f}$ has compact support. The function

$$
g(t, x)=g_{t}(x) \text { for } g_{t}=\mathrm{e}^{-\mathrm{i} t \omega\left(D_{x}\right)} f,
$$

which solves $\left(\partial_{t}^{2}-\Delta_{x}\right) g+m^{2} g=0$, will be called a positive energy KG solution.

The following property of positive energy KG solutions is proven in [RS3]:

Proposition 6.2. Let $\chi_{1}, \chi_{2} \in C^{\infty}\left(\mathbb{R}^{d}\right)$ be bounded with all derivatives and having disjoint supports. Let $f \in \mathcal{S}\left(\mathbb{R}^{d}\right)$ be s.t. $\widehat{f}$ has compact support. Then

$$
\left\|\chi_{1}\left(\frac{x}{t}\right) \mathrm{e}^{-\mathrm{i} t \omega\left(D_{x}\right)} \chi_{2}\left(\nabla \omega\left(D_{x}\right)\right) f\right\|_{L^{2}\left(\mathbb{R}^{d}\right)} \in O\left(t^{-\infty}\right) .
$$

We recall the notion of velocity support which will be useful later on.

Definition 6.3. Let $\Delta \Subset H_{m}$. We set

$$
\operatorname{Vel}(\Delta):=\left\{\nabla \omega(p): p \in \mathbb{R}^{d},(\omega(p), p) \in \Delta\right\} .
$$

It is clear that disjointness of $\Delta_{1}$ and $\Delta_{2}$ entails that $\operatorname{Vel}\left(\Delta_{1}\right)$ and $\operatorname{Vel}\left(\Delta_{2}\right)$ are also disjoint. In view of Proposition 6.2 and of the fact that supp $\widehat{g} \subset H_{m}$, we can call $\operatorname{Vel}(\operatorname{supp} \widehat{g})=\{\nabla \omega(p): p \in \operatorname{supp} \widehat{f}\}$ the velocity support of a positive energy $\mathrm{KG}$ solution $g$ with initial data $f$.

6.2. Haag-Ruelle scattering theory. Let $B \in \mathcal{L}_{0}$ satisfy (2.11), that is $-\operatorname{supp}(\widehat{B}) \cap$ $\mathcal{S} p U \subset H_{m}$, and let $g$ be a positive energy $\mathrm{KG}$ solution. The Haag-Ruelle creation operator is given by

$$
B_{t}^{*}\left(g_{t}\right)=\int g(t, x) B^{*}(t, x) d x, \quad t \in \mathbb{R},
$$

which is well defined since $\mathrm{e}^{-\mathrm{i} t \omega\left(D_{x}\right)}$ preserves $\mathcal{S}\left(\mathbb{R}^{d}\right)$. The following lemma is elementary, except for part (2) which relies on Lemma 3.4. We refer to [DG12] for a proof.

Lemma 6.4. The following properties hold:

(1) $B_{t}^{*}\left(g_{t}\right) \Omega=B^{*}(f) \Omega=(2 \pi)^{d / 2} \widehat{f}(P) B^{*} \Omega$, if $g_{t}=\mathrm{e}^{-\mathrm{i} t \omega\left(D_{x}\right)} f$.

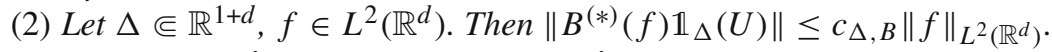

(3) $\partial_{t} B_{t}^{*}\left(g_{t}\right)=\dot{B}_{t}^{*}\left(g_{t}\right)+B_{t}^{*}\left(\dot{g}_{t}\right)$, where $\dot{B}=\partial_{s} B(s, 0)_{\mid s=0} \in \mathcal{L}_{0}$ and $\dot{g}=\partial_{t} g$ is a positive energy $K G$ solution with the same velocity support as $g$.

The following result is known as the Haag-Ruelle theorem [Ha58, Ru62]. In Appendix B.3 we give an elementary proof which uses ideas from [He65,BF82, Ar99, Dy05] and exploits the bound in Lemma 6.4 (2).

Theorem 6.5. Let $B_{1}, \ldots, B_{n} \in \mathcal{L}_{0}$ satisfy (2.11). Let $g_{1}, \ldots, g_{n}$ be positive energy $K G$ solutions with disjoint velocity supports. Then:

(1) There exists the $n$-particle scattering state given by

$$
\Psi^{+}=\lim _{t \rightarrow \infty} B_{1, t}^{*}\left(g_{1, t}\right) \ldots B_{n, t}^{*}\left(g_{n, t}\right) \Omega .
$$


(2) The state $\Psi^{+}$depends only on the single-particle vectors $\Psi_{i}=B_{i, t}^{*}\left(g_{i, t}\right) \Omega$, and therefore we can write $\Psi^{+}=\Psi_{1} \stackrel{\text { out }}{\times} \stackrel{\text { out }}{\times} \Psi_{n}$. Given two such vectors $\Psi^{+}$and $\tilde{\Psi}^{+}$ one has:

$$
\begin{aligned}
\left(\tilde{\Psi}^{+} \mid \Psi^{+}\right) & =\sum_{\sigma \in S_{n}}\left(\tilde{\Psi}_{1} \mid \Psi_{\sigma_{1}}\right) \ldots\left(\tilde{\Psi}_{n} \mid \Psi_{\sigma_{n}}\right), \\
U(t, x)\left(\Psi_{1} \stackrel{\text { out }}{\times} \cdots \times \stackrel{\text { out }}{\times} \Psi_{n}\right) & =\left(U(t, x) \Psi_{1}\right) \stackrel{\text { out }}{\times} \cdots \stackrel{\text { out }}{\times}\left(U(t, x) \Psi_{n}\right), \quad(t, x) \in \mathbb{R}^{1+d},
\end{aligned}
$$

where $S_{n}$ is the set of permutations of an n-element set.

Let us now explain how to obtain the (outgoing) n-particle wave operator: Let

$$
\mathcal{H}_{m}:=\mathbb{1}_{H_{m}}(U) \mathcal{H},
$$

be the space of one-particle states. For $\Psi_{1}, \ldots, \Psi_{n} \in \mathcal{H}_{m}$ we set

$$
\Psi_{1} \otimes_{\mathrm{s}} \cdots \otimes_{\mathrm{s}} \Psi_{n}:=\frac{1}{\sqrt{n !}} \sum_{\sigma \in S_{n}} \Psi_{\sigma_{1}} \otimes \cdots \otimes \Psi_{\sigma_{n}} \in \mathcal{H}_{m}^{\otimes_{\mathrm{s}} n} .
$$

Proposition 6.6. For any $n \geq 1$ there exists a unique isometry

$$
W_{n}^{+}: \mathcal{H}_{m}^{\otimes_{s} n} \rightarrow \mathcal{H}
$$

with the following properties:

(1) If $\Psi_{1}, \ldots, \Psi_{n}$ are as in Theorem 6.5 , then $W_{n}^{+}\left(\Psi_{1} \otimes_{\mathrm{s}} \cdots \otimes_{\mathrm{s}} \Psi_{n}\right)=\Psi_{1} \times \stackrel{\text { out }}{\times} \stackrel{\text { out }}{\times} \Psi_{n}$.

(2) $U(t, x) \circ W_{n}^{+}=W_{n}^{+} \circ\left(U_{m}(t, x) \otimes \cdots \otimes U_{m}(t, x)\right),(t, x) \in \mathbb{R}^{1+d}$, where we denote by $U_{m}(t, x)$ the restriction of $U(t, x)$ to $\mathcal{H}_{m}$.

Definition 6.7. Let $W_{n}^{+}, n \geq 1$, be the isometries defined in Proposition 6.6 and let $u s$ define $W_{0}^{+}: \mathbb{C} \Omega \rightarrow \mathcal{H}$ by $W_{0}^{+} \Omega=\Omega$. Let $\Gamma\left(\mathcal{H}_{m}\right)$ be the symmetric Fock space over $\mathcal{H}_{m}$ and let $W^{+}: \Gamma\left(\mathcal{H}_{m}\right) \rightarrow \mathcal{H}$ be the isometry given by $W^{+}:=\bigoplus_{n \geq 0} W_{n}^{+}$.

(1) The map $W_{n}^{+}: \mathcal{H}_{m}^{\otimes_{\mathrm{s}} n} \rightarrow \mathcal{H}$ is called the (outgoing) $n$-particle wave operator.

(2) The map $W^{+}: \Gamma\left(\mathcal{H}_{m}\right) \rightarrow \mathcal{H}$ is called the (outgoing) wave operator.

(3) The range of $W_{n}^{+}$is denoted by $\mathcal{H}_{n}^{+}$and called the subspace of $n$-particle scattering states.

(4) The range of $W^{+}$is denoted by $\mathcal{H}^{+}$and called the subspace of scattering states.

Proof of Proposition 6.6. Let $\mathcal{F} \subset \mathcal{H}_{m}^{\otimes_{s} n}$ be the subspace spanned by vectors $\Psi_{1} \otimes_{\mathrm{s}}$ $\cdots \otimes_{\mathrm{s}} \Psi_{n}$ for $\Psi_{1}, \ldots, \Psi_{n}$ as in Theorem 6.5. Due to (6.2) there exists a unique isometry $W_{n}^{+}: \mathcal{F} \rightarrow \mathcal{H}$ such that

$$
W_{n}^{+}\left(\Psi_{1} \otimes_{\mathrm{s}} \ldots \otimes_{\mathrm{s}} \Psi_{n}\right)=\Psi_{1} \stackrel{\text { out }}{\times} \ldots \stackrel{\text { out }}{\times} \Psi_{n},
$$

for all $\Psi_{1}, \ldots, \Psi_{n}$ as in the theorem. Also, by (6.3),

$$
U(t, x) \circ W_{n}^{+}=W_{n}^{+} \circ\left(U_{m}(t, x) \otimes \cdots \otimes U_{m}(t, x)\right) .
$$

Thus it suffices to prove that the closure of $\mathcal{F}$ is $\mathcal{H}_{m}^{\otimes_{\mathrm{s}} n}$. 
Let $\left(H_{i}, P_{i}\right), i=1, \ldots, n$, be the generators of the groups of unitaries

$$
(t, x) \mapsto \underbrace{\mathbb{1} \otimes \cdots \otimes \mathbb{1}}_{i-1} \otimes U_{m}(t, x) \otimes \underbrace{\mathbb{1} \otimes \cdots \otimes \mathbb{1}}_{n-i},
$$

acting on $\mathcal{H}_{m}^{\otimes n}$. We note that the joint spectral measure of $(\tilde{H}, \tilde{P}):=\left(\left(H_{1}, P_{1}\right), \ldots\right.$, $\left.\left(H_{n}, P_{n}\right)\right)$ is supported by $H_{m}^{\times n}$.

Let $B \in \mathcal{L}_{0}$ satisfy (2.11) and $g$ be a positive energy $\mathrm{KG}$ solution. Then, due to Lemma 6.4 (1) and the cyclicity of the vacuum, the set of vectors $B_{t}^{*}\left(g_{t}\right) \Omega$ is dense in $\mathcal{H}_{m}$. Also, for $\Delta \Subset H_{m}$, the set of such vectors with $g$ having the velocity support included in $\operatorname{Vel}(\Delta)$ is dense in $\mathbb{1}_{\Delta}(U) \mathcal{H}_{m}$. Thus the closure of $\mathcal{F}$ in $\mathcal{H}_{m}^{\otimes_{s} n}$ equals

$$
\mathcal{F}^{\mathrm{cl}}=\Theta_{\mathrm{s}} \circ \mathbb{1}_{\left(H_{m}^{\times n}\right) \backslash D}(\tilde{H}, \tilde{P}) \mathcal{H}_{m}^{\otimes n},
$$

where $\Theta_{\mathrm{s}}: \mathcal{H}_{m}^{\otimes n} \rightarrow \mathcal{H}_{m}^{\bigotimes_{\mathrm{s}} n}$ is the orthogonal projection, and

$$
D:=\left\{\underline{p} \in H_{m}^{\times n}: p_{i}=p_{j} \text { for some } i \neq j\right\} .
$$

By [BF82, Prop. 2.2], the spectral measure of the restriction of $(H, P)$ to $\mathcal{H}_{m}$ is absolutely continuous w.r.t. the Lorentz invariant measure on $H_{m}$. Hence $\mathbb{1}_{D}(\tilde{H}, \tilde{P})=0$, which completes the proof.

\section{Proof of Theorem 2.9}

We recall that the notation $N_{B}\left(h_{1}\right), N_{B}\left(h_{2}^{\mathrm{w}}, t\right)$ was introduced in Definition 3.7 and in (4.1), respectively, for $B \in \mathcal{L}_{0}, h_{1} \in B\left(L^{2}\left(\mathbb{R}^{d}\right)\right)$ and $h_{2} \in \mathcal{S}\left(T^{*} \mathbb{R}^{d}\right)$.

Proposition 7.1. Let $i=1, \ldots, n$. Let $\Delta_{i} \Subset H_{m}$ be disjoint sets, $B_{i} \in \mathcal{L}_{0}$ and $\operatorname{supp}\left(\widehat{B}_{i}\right)$ be disjoint sets. Assume moreover that:

$$
\begin{array}{r}
-\operatorname{supp}\left(\widehat{B}_{i}\right) \cap \mathcal{S} p U \subset \Delta_{i}, \\
\left(\Delta_{i}+\operatorname{supp}\left(\widehat{B}_{i}\right)\right) \cap \mathcal{S} p(U) \subset\{0\} .
\end{array}
$$

Let $h_{i} \in C_{0}^{\infty}\left(T^{*} \mathbb{R}^{d}\right)$ such that

$$
\begin{aligned}
& \text { (1) } h_{i}\left(y,(\nabla \omega)^{-1}(y)\right)=1, y \in \operatorname{Vel}\left(\Delta_{i}\right) \text {, } \\
& \text { (2) } \pi_{x} \operatorname{supp} h_{i} \cap \pi_{x} \operatorname{supp} h_{j}=\emptyset, \forall i \neq j .
\end{aligned}
$$

Then, for $\Psi_{i} \in \mathbb{1}_{\Delta_{i}}(U) \mathcal{H}$,

$$
\begin{aligned}
& \lim _{t \rightarrow+\infty} N_{B_{1}}\left(h_{1}^{\mathrm{w}}, t\right) \ldots N_{B_{n}}\left(h_{n}^{\mathrm{w}}, t\right) W_{n}^{+}\left(\Psi_{1} \otimes_{\mathrm{s}} \cdots \otimes_{\mathrm{s}} \Psi_{n}\right) \\
& \quad=W_{n}^{+}\left(N_{B_{1}}(\mathbb{1}) \Psi_{1} \otimes_{\mathrm{s}} \cdots \otimes_{\mathrm{s}} N_{B_{n}}(\mathbb{1}) \Psi_{n}\right) .
\end{aligned}
$$

Remark 7.2. Note that $W_{n}^{+}\left(\Psi_{1} \otimes_{\mathrm{s}} \cdots \otimes_{\mathrm{s}} \Psi_{n}\right)$ belongs to $\mathcal{H}_{\mathrm{c}}(U)$, and that $N_{B_{i}}(\mathbb{1}) \Psi_{i}$ belong to $\mathbb{1}_{\Delta_{i}}(U) \mathcal{H}$, because of (7.1), (7.2). Hence all the expressions appearing in (7.4) are well defined. 
Proof. Due to the fact that $N_{B_{i}}(\mathbb{1}) \Psi_{i}$ satisfy the assumption imposed on $\Psi_{i}$ in the proposition, it suffices to show that

$\lim _{t \rightarrow+\infty} N_{B_{i}}\left(h_{i}^{\mathrm{w}}, t\right) W_{n}^{+}\left(\Psi_{1} \otimes_{\mathrm{s}} \cdots \otimes_{\mathrm{s}} \Psi_{n}\right)=W_{n}^{+}\left(\Psi_{1} \otimes_{\mathrm{s}} \cdots \otimes_{\mathrm{s}} N_{B_{i}}(\mathbb{1}) \Psi_{i} \otimes_{\mathrm{s}} \cdots \otimes_{\mathrm{s}} \Psi_{n}\right)$

and then iterate this result making use of the bound $\sup _{t \in \mathbb{R}}\left\|N_{B_{i}}\left(h_{i}^{\mathrm{w}}, t\right) \mathbb{1}_{\Delta}(U)\right\|<\infty$ valid for any $\Delta \Subset \mathbb{R}^{1+d}$. By the same token, it suffices to assume that $\Psi_{j}=A_{j, t}^{*}\left(g_{j, t}\right) \Omega$ for $A_{j} \in \mathcal{L}_{0}$ satisfying (7.1) and $g_{j}$ a positive energy KG solution with the velocity support included in $\operatorname{Vel}\left(\Delta_{j}\right)$, so that $\Psi_{j}=\mathbb{1}_{\Delta_{j}}(U) \Psi_{j}$. Similarly, since $N_{B_{i}}(\mathbb{1}) \Psi_{i} \in$ $\mathbb{1}_{\Delta_{i}}(U) \mathcal{H}$, we can find for any $0<\epsilon_{i} \ll 1$ operators $\tilde{A}_{i} \in \mathcal{L}_{0}$ and positive energy KG solutions $\tilde{g}_{i}$, satisfying the same properties as $A_{i}, g_{i}$, such that

$$
\left\|N_{B_{i}}(\mathbb{1}) \Psi_{i}-\tilde{A}_{i, t}^{*}\left(\tilde{g}_{i, t}\right) \Omega\right\| \leq \epsilon_{i}, \quad i=1, \ldots, n .
$$

We fix such $A_{j}, g_{j}$ and $\tilde{A}_{i}, \tilde{g}_{i}$ for future reference.

First, we claim that for $B, \Delta, \Psi, h$ as in the proposition one has:

$$
\lim _{t \rightarrow+\infty} N_{B}\left(h^{\mathrm{w}}, t\right) \Psi=N_{B}(\mathbb{1}) \Psi .
$$

In fact, due to (7.1), (7.2) we have

$$
B^{*} B \mathbb{1}_{\Delta}(U)=B^{*}|\Omega\rangle\langle\Omega| B \mathbb{1}_{\Delta}(U)=\mathbb{1}_{\Delta}(U) B^{*} B \mathbb{1}_{\Delta}(U) .
$$

Therefore,

$$
\begin{aligned}
N_{B}\left(h^{\mathrm{w}}, t\right) \Psi & =\mathrm{e}^{\mathrm{i} t H} N_{B}\left(h_{t}^{\mathrm{w}}\right) \mathrm{e}^{-\mathrm{i} t H} \Psi \\
& =\mathrm{e}^{\mathrm{i} t \omega(P)} a_{B}^{*} \circ\left(\mathbb{1}_{\mathcal{H}} \otimes h_{t}^{\mathrm{w}}\right) \circ a_{B} \mathrm{e}^{-\mathrm{i} t \omega(P)} \Psi \\
& =a_{B}^{*} \circ \mathrm{e}^{\mathrm{i} t \omega\left(P+D_{x}\right)}\left(\mathbb{1}_{\mathcal{H}} \otimes h_{t}^{\mathrm{w}}\right) \mathrm{e}^{-\mathrm{i} t \omega\left(P+D_{x}\right)} \circ a_{B} \Psi \\
& =a_{B}^{*} \circ\left(\mathbb{1}_{\mathcal{H}} \otimes \mathrm{e}^{\mathrm{i} t \omega\left(D_{x}\right)} h_{t}^{\mathrm{w}} \mathrm{e}^{-\mathrm{i} t \omega\left(D_{x}\right)}\right) \circ a_{B} \Psi,
\end{aligned}
$$

where we used (3.8) and the fact that $a_{B} \Psi=\left(|\Omega\rangle\langle\Omega| \otimes \mathbb{1}_{L^{2}\left(\mathbb{R}^{d}\right)}\right) \circ a_{B} \Psi$. Making use of Proposition 3.1 (2), we get

$$
\mathrm{s}-\lim _{t \rightarrow \infty} \mathrm{e}^{\mathrm{i} t \omega\left(D_{x}\right)} h_{t}^{\mathrm{w}} \mathrm{e}^{-\mathrm{i} t \omega\left(D_{x}\right)}=h\left(\nabla \omega\left(D_{x}\right), D_{x}\right) .
$$

Thus we obtain

$$
\lim _{t \rightarrow+\infty} N_{B}\left(h^{\mathrm{w}}, t\right) \Psi=a_{B}^{*} \circ\left(\mathbb{1}_{\mathcal{H}} \otimes h\left(\nabla \omega\left(D_{x}\right), D_{x}\right)\right) \circ a_{B} \Psi=a_{B}^{*} a_{B} h(\nabla \omega(P), P) \Psi,
$$

exploiting Lemma 3.5 and once again (3.8). By (7.3) (1) we have $h(\nabla \omega(p), p)=1$ for $(\omega(p), p) \in \Delta$. Hence $h(\nabla \omega(P), P) \Psi=\Psi$, which completes the proof of (7.7).

Next, we claim that for $i \neq j$ :

$$
\left\|\left[N_{B_{i}}\left(h_{i}^{\mathrm{W}}, t\right), A_{j, t}^{*}\left(g_{j, t}\right)\right]\right\| \in O\left(t^{-\infty}\right) .
$$

To show (7.11), we first note that $\operatorname{Vel}\left(\Delta_{j}\right) \subset \pi_{x} \operatorname{supp} h_{j}$ by (7.3) (1). Hence $\pi_{x} \operatorname{supp} h_{i}$ and the velocity support of $g_{j}$ are disjoint by (7.3) (2). Let $\chi_{i}, \chi_{j} \in C_{0}^{\infty}\left(\mathbb{R}^{d}\right)$ with $0 \leq \chi_{i}, \chi_{j} \leq 1, \operatorname{supp} \chi_{i} \cap \operatorname{supp} \chi_{j}=\emptyset$ and $\chi_{i} \equiv 1$ near $\pi_{x} \operatorname{supp} h_{i}, \chi_{j} \equiv 1$ near the velocity support of $g_{j}$. We set $\chi_{i, t}(x):=\chi_{i}(x / t), \chi_{j, t}(x):=\chi_{j}(x / t)$ and denote the 
corresponding operators on $L^{2}\left(\mathbb{R}^{d}\right)$ by the same symbols. We recall that $g_{N}$ is defined in (3.12) and note that

$$
\left\|\left(1-\chi_{i, t}\right) h_{i, t}^{\mathrm{w}}\right\|_{B\left(L^{2}\left(\mathbb{R}^{d}\right)\right)},\left\|h_{i, t}^{\mathrm{w}}\left(1-\chi_{i, t}\right)\right\|_{B\left(L^{2}\left(\mathbb{R}^{d}\right)\right)},\left\|\chi_{i, t} g_{N}\left(D_{x}\right) \chi_{j, t}\right\|_{B\left(L^{2}\left(\mathbb{R}^{d}\right)\right)} \in O\left(t^{-\infty}\right),
$$

where the expressions involving $h_{i, t}^{\mathrm{w}}$ are treated using Proposition 3.1 (6) and the expression with $g_{N}$ by inspection of its kernel as in the proof of Corollary 3.13. By Proposition 6.2 we have:

$$
\left\|\left(1-\chi_{j, t}\right) g_{j, t}\right\|_{L^{2}\left(\mathbb{R}^{d}\right)} \in O\left(t^{-\infty}\right) .
$$

Then (7.11) follows by applying Lemma 3.14 for $B_{1}=B_{i}, B_{2}=A_{j}, h_{1}=h_{i, t}^{\mathrm{w}}$, $g_{2}=g_{j, t}$ and $\chi_{1}=\chi_{i, t}, \chi_{2}=\chi_{j, t}$. Indeed, the quantities in the r.h.s. of (3.14) are $O\left(t^{-\infty}\right)$ by (7.12) and (7.13).

After these preparations we proceed to the proof of (7.5). Using (7.11), (7.7), we obtain:

$$
\begin{aligned}
N_{B_{i}}\left(h_{i}^{\mathrm{w}}, t\right)\left(\Psi_{1} \times \stackrel{\text { out }}{\times} \cdots \stackrel{\text { out }}{\times} \Psi_{n}\right) & =N_{B_{i}}\left(h_{i}^{\mathrm{w}}, t\right) A_{1, t}^{*}\left(g_{1, t}\right) \ldots A_{n, t}^{*}\left(g_{n, t}\right) \Omega+o\left(t^{0}\right) \\
& =A_{1, t}^{*}\left(g_{1, t}\right) \ldots \check{i} \ldots A_{n, t}^{*}\left(g_{n, t}\right) N_{B_{i}}(\mathbb{1}) \Psi_{i}+o\left(t^{0}\right) \\
& =A_{1, t}^{*}\left(g_{1, t}\right) \ldots \check{i} \ldots A_{n, t}^{*}\left(g_{n, t}\right) \tilde{A}_{i, t}^{*}\left(\tilde{g}_{i, t}\right) \Omega+o\left(t^{0}\right)+O\left(t^{0}\right) \epsilon_{i} \\
& =\Psi_{1} \stackrel{\text { out }}{\times} \ldots \stackrel{\text { out }}{\times} \tilde{\Psi}_{i} \stackrel{\text { out } \times \text { out }}{\times} \Psi_{n}+o\left(t^{0}\right)+O\left(t^{0}\right) \epsilon_{i} \\
& =\Psi_{1} \stackrel{\text { out }}{\times} \cdots \stackrel{\text { out }}{\times} N_{B_{i}}(\mathbb{1}) \Psi_{i} \stackrel{\text { out }}{\times} \ldots \stackrel{\text { out }}{\times} \Psi_{n}+o\left(t^{0}\right)+O\left(t^{0}\right) \epsilon_{i},
\end{aligned}
$$

where $\tilde{\Psi}_{i}:=\tilde{A}_{i, t}^{*}\left(\tilde{g}_{i, t}\right) \Omega$. Since $\epsilon_{i}>0$ is arbitrary, this concludes the proof of the proposition.

Lemma 7.3. Let $\Delta \subset G_{2 m}$ be an open bounded set. Then

$$
\begin{gathered}
\mathbb{1}_{\Delta}(U) \mathcal{H}_{n}^{+}=\operatorname{Span}\left\{W_{n}^{+}\left(\Psi_{1} \otimes_{\mathrm{s}} \cdots \otimes_{\mathrm{s}} \Psi_{n}\right): \Psi_{i} \in \mathbb{1}_{\Delta_{i}}(U) \mathcal{H}, \Delta_{i} \Subset H_{m},\right. \\
\left.\Delta_{i} \cap \Delta_{j}=\emptyset, i \neq j, \Delta_{1}+\cdots+\Delta_{n} \subset \Delta\right\}^{\mathrm{cl}} .
\end{gathered}
$$

Proof. The statement follows immediately from Proposition 6.6 (2) and the absolute continuity of the spectral measure of $(H, P)$ restricted to $\mathcal{H}_{m}$, recalled in its proof.

Lemma 7.4. Let $\Delta \subset G_{2 m}$ be an open bounded set s.t. $(\bar{\Delta}-\bar{\Delta}) \cap \mathcal{S} p U=\{0\}$. Let $\Delta_{1}, \ldots, \Delta_{n} \Subset H_{m}$ be disjoint and such that $\Delta_{1}+\cdots+\Delta_{n} \subset \Delta$. Then there exist $O_{1}, \ldots, O_{n} \subset \mathbb{R}^{1+d}$ which are disjoint open neighbourhoods of $\Delta_{1}, \ldots, \Delta_{n}$, respectively, s.t. for any $K_{1}, \ldots, K_{n} \Subset \mathbb{R}^{1+d}$ satisfying $-K_{i} \subset O_{i},-K_{i} \cap \mathcal{S} p U \subset \Delta_{i}$, $i=1, \ldots, n$, one has:

$$
\begin{aligned}
&\left(\bar{\Delta}+K_{1}+\cdots+K_{n}\right) \cap \mathcal{S} p U \subset\{0\}, \\
&-\left(K_{1}+\cdots+K_{n}\right) \subset \Delta, \\
&\left(\Delta_{i}+K_{i}\right) \cap \mathcal{S} p U \subset\{0\} .
\end{aligned}
$$

Proof. Assume that $O_{i} \subset \Delta_{i}+\mathcal{B}_{1}(0, \varepsilon)$, where $\mathcal{B}_{1}(0, \varepsilon):=\left\{x \in \mathbb{R}^{d}:|x|<\varepsilon\right\}$. To prove (7.14), we write

$$
\begin{aligned}
\bar{\Delta}+K_{1}+\cdots+K_{n} \subset \bar{\Delta}-\left(O_{1}+\cdots+O_{n}\right) & \subset \bar{\Delta}-\left(\Delta_{1}+\cdots+\Delta_{n}\right)+\mathcal{B}_{1}(0, n \varepsilon) \\
& \subset \bar{\Delta}-\bar{\Delta}+\mathcal{B}_{1}(0, n \varepsilon) .
\end{aligned}
$$


Since, by assumption, $(\bar{\Delta}-\bar{\Delta}) \cap \mathcal{S} p U=\{0\}$ and 0 is isolated in $\mathcal{S} p U$, we obtain that $\left(\bar{\Delta}-\bar{\Delta}+\mathcal{B}_{1}(0, n \varepsilon)\right) \cap \mathcal{S} p U=\{0\}$ for $\varepsilon \ll 1$. As for (7.15), we obtain that

$$
-\left(K_{1}+\cdots+K_{n}\right) \subset O_{1}+\cdots+O_{n} \subset \Delta_{1}+\cdots+\Delta_{n}+\mathcal{B}_{1}(0, n \varepsilon) \subset \Delta,
$$

for $\varepsilon \ll 1$ using that $\Delta_{i}$ are compact and $\Delta$ is open. Finally we write:

$$
\Delta_{i}+K_{i} \subset O_{i}-O_{i} \subset \Delta_{i}-\Delta_{i}+\mathcal{B}_{1}(0,2 \varepsilon) .
$$

We note that a difference of two vectors from $H_{m}$ is either 0 or spacelike. For $\varepsilon \ll 1$ we obtain (7.16).

Lemma 7.5. Let $\Delta \Subset H_{m}$ and $O \subset \mathbb{R}^{1+d}$ be a sufficiently small neighbourhood of $\Delta$. Then

$$
\begin{aligned}
& \mathbb{1}_{\Delta}(U) \mathcal{H}=\operatorname{Span}\{ N_{B}\left(\mathbb{1}^{\prime} \mathbb{1}_{\Delta}(U) \mathcal{H}: B \in \mathcal{L}_{0},\right. \\
&-\operatorname{supp}(\widehat{B}) \subset O,-\operatorname{supp}(\widehat{B}) \cap \mathcal{S} p U \subset \Delta\}^{\mathrm{cl}} .
\end{aligned}
$$

Proof. A proof of this lemma, which is based on ideas from [DT11a, Thm. 3.5], can be found in [DG12].

Proof of Theorem 2.9. By Theorem 2.8, it is enough to verify that

$$
\mathbb{1}_{\Delta}(U) \mathcal{H}_{n}^{+} \subset \operatorname{Span}\left\{\operatorname{Ran} Q_{n, \alpha}^{+}(\Delta): \alpha \in J\right\}^{\mathrm{cl}} .
$$

In view of Lemma 7.3, it suffices to show that for any $\Delta_{i} \Subset H_{m}, i=1, \ldots, n$, such that $\Delta_{1}+\cdots+\Delta_{n} \subset \Delta$ and $\Delta_{i} \cap \Delta_{j}=\emptyset$ for $i \neq j$ one has

$$
W_{n}^{+}\left(\mathbb{1}_{\Delta_{1}}(U) \mathcal{H} \otimes_{\mathrm{s}} \cdots \otimes_{\mathrm{s}} \mathbb{1}_{\Delta_{n}}(U) \mathcal{H}\right) \subset \operatorname{Span}\left\{\operatorname{Ran} Q_{n, \alpha}^{+}(\Delta): \alpha \in J\right\}^{\mathrm{cl}} .
$$

Let $O_{i} \subset \mathbb{R}^{1+d}$ be sufficiently small open neighbourhoods of $\Delta_{i}$ so that the assertions of Lemma 7.4 hold. Let $B_{i} \in \mathcal{L}_{0}$ be such that $-\operatorname{supp}\left(\widehat{B}_{i}\right) \subset O_{i},-\operatorname{supp}\left(\widehat{B}_{i}\right) \cap \mathcal{S} p U \subset$ $\Delta_{i}$. By Lemma 7.4, $B_{i}$ are $\Delta$-admissible in the sense of Definition 2.5 and satisfy the assumptions of Proposition 7.1. We also choose $h_{i} \in C_{0}^{\infty}\left(T^{*} \mathbb{R}^{d}\right)$ as in Proposition 7.1 and s.t. $h_{1} \otimes \cdots \otimes h_{n}$ is admissible in the sense of Definition 2.7. For example one can choose $h_{i}(x, \xi):=h_{0, i}(x) \chi(x-\nabla \omega(\xi))$, where $h_{0, i} \in C_{0}^{\infty}\left(\mathbb{R}^{d}\right)$ are equal to one on $\operatorname{Vel}\left(\Delta_{i}\right)$ and have disjoint supports contained in the unit ball. The function $\chi \in C_{0}^{\infty}\left(\mathbb{R}^{d}\right)$ satisfies $\chi(0)=1$ and is supported in a sufficiently small neighbourhood of zero, depending on the supports of $h_{0, i}$.

Let $J_{0}$ be the set of pairs $(\underline{B}, \underline{h})$ as specified above. We get

$$
\begin{aligned}
& \operatorname{Span}\left\{Q_{n, \alpha}^{+}(\Delta) \circ W_{n}^{+}\left(\mathbb{1}_{\Delta_{1}}(U) \mathcal{H} \otimes_{\mathrm{s}} \cdots \otimes_{\mathrm{s}} \mathbb{1}_{\Delta_{n}}(U) \mathcal{H}\right): \alpha \in J_{0}\right\} \\
& \quad=\operatorname{Span}\left\{W_{n}^{+}\left(N_{B_{1}}\left(\mathbb{1}_{1} \mathbb{1}_{\Delta_{1}}(U) \mathcal{H} \otimes_{\mathrm{s}} \cdots \otimes_{\mathrm{s}} N_{B_{n}}(\mathbb{1}) \mathbb{1}_{\Delta_{n}}(U) \mathcal{H}\right): \alpha \in J_{0}\right\}\right. \\
& =W_{n}^{+}\left(\mathbb{1}_{\Delta_{1}}(U) \mathcal{H} \otimes_{\mathrm{s}} \cdots \otimes_{\mathrm{s}} \mathbb{1}_{\Delta_{n}}(U) \mathcal{H}\right) .
\end{aligned}
$$

In the first step we used Proposition 7.1 and in the second Lemma 7.5. Since $J_{0} \subset J$, the subspace on the 1.h.s. of (7.19) is included in the subspace on the r.h.s. of (7.18). This concludes the proof.

Acknowledgements. W. D. would like to thank D. Buchholz, W. De Roeck, J. Fröhlich, G. M. Graf, A. Jaffe and J. S. Møller for interesting discussions. Financial support of the German Research Foundation (DFG) within the stipend DY107/1-1 is gratefully acknowledged. 


\section{Appendix A: Propagation Estimates for Inhomogeneous Evolution Equations}

In this section, which appeared already in [DG12], we extend standard results on propagation estimates and existence of asymptotic observables to the case of an inhomogeneous evolution equation:

$$
\partial_{t} u(t)=-\mathrm{i} H u(t)+r(t) .
$$

Let $\mathcal{H}$ be a Hilbert space and $H$ a self-adjoint operator on $\mathcal{H}$. We choose a function

$$
\mathbb{R}^{+} \ni t \mapsto u(t) \in \mathcal{H},
$$

such that

$$
\begin{aligned}
& \text { (i) } \sup _{t \geq 0}\|u(t)\|<\infty, \\
& \text { (ii) } u(t) \in C^{1}\left(\mathbb{R}^{+}, \mathcal{H}\right) \cap C^{0}\left(\mathbb{R}^{+} \text {, Dom } H\right) \text {, }
\end{aligned}
$$

and define:

$$
r(t):=\partial_{t} u(t)+\mathrm{i} H u(t) .
$$

For a map $\mathbb{R}^{+} \ni t \mapsto M(t) \in B(\mathcal{H})$ we denote by $\mathcal{D} M(t)=\partial_{t} M(t)+[H, \mathrm{i} M(t)]$ the Heisenberg derivative of $M(t)$. We assume that $[H, \mathrm{i} M(t)]$, defined first as a quadratic form on Dom $H$, extends by continuity to a bounded operator.

The following three lemmas can be proved by modifying standard arguments, see e.g. [DG97, Sect. B.4]. By $C_{j}(\cdot), B(\cdot), B_{1}(\cdot)$ we denote auxiliary functions from $\mathbb{R}^{+}$ to $B(\mathcal{H})$.

Lemma A.1. Let $\mathbb{R}^{+} \ni t \mapsto M(t) \in B(\mathcal{H})$ be s.t.:

(i) $\sup _{t \in \mathbb{R}^{+}}\|M(t)\|<\infty, \quad\|M(\cdot) r(\cdot)\|,\left\|M^{*}(\cdot) r(\cdot)\right\| \in L^{1}\left(\mathbb{R}^{+}, d t\right)$,

(ii) $\mathcal{D} M(t) \geq B^{*}(t) B(t)-\sum_{j=1}^{n} C_{j}^{*}(t) C_{j}(t), \int_{\mathbb{R}^{+}}\left\|C_{j}(t) u(t)\right\|^{2} d t<\infty$.

Then

$$
\int_{\mathbb{R}^{+}}\|B(t) u(t)\|^{2} d t<\infty .
$$

Lemma A.2. Let $\mathbb{R}^{+} \ni t \mapsto M(t) \in B(\mathcal{H})$ be s.t.:

$$
\begin{aligned}
& \text { (i) } \sup _{t \in \mathbb{R}^{+}}\|M(t)\|<\infty, \quad\|M(\cdot) r(\cdot)\|,\left\|M^{*}(\cdot) r(\cdot)\right\| \in L^{1}\left(\mathbb{R}^{+}, d t\right), \\
& \text { (ii) }\left|\left(u_{1} \mid \mathcal{D} M(t) u_{2}\right)\right| \leq \sum_{j=1}^{n}\left\|C_{j}(t) u_{1}\right\|\left\|C_{j}(t) u_{2}\right\|, u_{1}, u_{2} \in \mathcal{H}, \\
& \text { with } \int_{\mathbb{R}^{+}}\left\|C_{j}(t) u(t)\right\|^{2} d t<\infty .
\end{aligned}
$$

Then

$$
\lim _{t \rightarrow+\infty}(u(t) \mid M(t) u(t)) \text { exists. }
$$


Lemma A.3. Let $\mathbb{R}^{+} \ni t \mapsto M(t) \in B(\mathcal{H})$ be s.t.:

(i) $\|M(\cdot) r(\cdot)\| \in L^{1}\left(\mathbb{R}^{+}, d t\right)$,

(ii) $\left|\left(u_{1} \mid \mathcal{D} M(t) u(t)\right)\right| \leq\left\|B_{1}(t) u_{1}\right\|\|B(t) u(t)\|$, with

(iii) $\int_{\mathbb{R}^{+}}\|B(t) u(t)\|^{2} d t<\infty, \int_{\mathbb{R}^{+}}\left\|B_{1}(t) \mathrm{e}^{-\mathrm{i} t H} u_{1}\right\|^{2} d t \leq C\left\|u_{1}\right\|^{2}, u_{1} \in \mathcal{H}$.

Then

$$
\lim _{t \rightarrow+\infty} \mathrm{e}^{\mathrm{i} t H} M(t) u(t) \text { exists. }
$$

\section{Appendix B: Some Technical Proofs}

In this section we give the proofs of Proposition 3.11, Lemma 3.14, Proposition 3.1 and Theorem 6.5 .

\section{B.1: Proof of Proposition 3.11.}

Proof. We will prove the proposition by induction on $n$. We set $\underline{x}=\left(x_{1}, \ldots, x_{n}\right), \underline{y}=$ $\left(y_{1}, \ldots, y_{n}\right) \in \mathbb{R}^{n d}$ and denote by $h_{i}\left(x_{i}, y_{i}\right)$ the distributional kernel of $h_{i}$.

We will also write $\tilde{h}_{i}=h_{i} \chi_{i}$ for $1 \leq i \leq n-1$ and $\tilde{h}_{n}=\chi_{n} h_{n}$. Note that

$$
\begin{aligned}
\tilde{h}_{i}\left(x_{i}, y_{i}\right) & =h_{i}\left(x_{i}, y_{i}\right) \chi_{i}\left(y_{i}\right), \quad 1 \leq i \leq n-1, \\
\tilde{h}_{n}\left(x_{n}, y_{n}\right) & =\chi_{n}\left(x_{n}\right) h_{n}\left(x_{n}, y_{n}\right) .
\end{aligned}
$$

We will first estimate the analog of $R_{n}$ with $h_{i}$ replaced with $\tilde{h}_{i}$, which will be denoted by $\tilde{R}_{n}$. Note first that since $B_{i}$ have compact energy-momentum transfers, for any $\Delta \Subset \mathbb{R}^{1+d}$ there exists $\Delta^{\prime} \Subset \mathbb{R}^{1+d}$ such that:

$$
\tilde{R}_{n} \mathbb{1}_{\Delta}(U)=\mathbb{1}_{\Delta^{\prime}}(U) \tilde{R}_{n} \mathbb{1}_{\Delta}(U),
$$

and therefore it suffices to estimate $\mathbb{1}_{\Delta_{1}}(U) \tilde{R}_{n} \mathbb{1}_{\Delta_{2}}(U)$ for $\Delta_{i} \Subset \mathbb{R}^{1+d}, i=1,2$.

Writing

$$
\tilde{R}_{n}=\int\left(\prod_{i=1}^{n} B_{i}^{*}\left(x_{i}\right) \prod_{i=1}^{n} B_{i}\left(y_{i}\right)-\prod_{i=1}^{n} B_{i}^{*}\left(x_{i}\right) B_{i}\left(y_{i}\right)\right) \prod_{i=1}^{n} \tilde{h}_{i}\left(x_{i}, y_{i}\right) d \underline{x} d \underline{y},
$$

and commuting $B_{n}^{*}\left(x_{n}\right)$ to the right, we obtain

$$
\tilde{R}_{n}=\tilde{R}_{n-1} \circ N_{B_{n}}\left(\tilde{h}_{n}\right)+\sum_{l=1}^{n-1} S_{n, l}
$$

for:

$$
S_{n, l}=\int\left(\prod_{i=1}^{n-1} B_{i}^{*}\left(x_{i}\right) \prod_{i=1}^{l-1} B_{i}\left(y_{i}\right)\left[B_{n}^{*}\left(x_{n}\right), B_{l}\left(y_{l}\right)\right] \prod_{i=l+1}^{n} B_{i}\left(y_{i}\right)\right) \prod_{i=1}^{n} \tilde{h}_{i}\left(x_{i}, y_{i}\right) d \underline{x} d \underline{y},
$$


where $\prod_{i=1}^{0} B_{i}\left(y_{i}\right)=1$ is understood. This implies that:

$$
\begin{aligned}
\left\|\mathbb{1}_{\Delta_{1}}(U) \tilde{R}_{n} \mathbb{1}_{\Delta_{2}}(U)\right\|_{B(\mathcal{H}) \leq} & \left\|\mathbb{1}_{\Delta_{1}}(U) \tilde{R}_{n-1} \mathbb{1}_{\Delta_{3}}(U)\right\|_{B(\mathcal{H})}\left\|\mathbb{1}_{\Delta_{3}}(U) N_{B_{n}}\left(\tilde{h}_{n}\right) \mathbb{1}_{\Delta_{2}}(U)\right\|_{B(\mathcal{H})} \\
& +\sum_{l=1}^{n-1}\left\|\mathbb{1}_{\Delta_{1}}(U) S_{n, l} \mathbb{1}_{\Delta_{2}}(U)\right\|_{B(\mathcal{H})} \\
\leq & C\left(B_{n}\right)\left\|\mathbb{1}_{\Delta_{1}}(U) \tilde{R}_{n-1} \mathbb{1}_{\Delta_{3}}(U)\right\|_{B(\mathcal{H})}\left\|h_{n}\right\|_{B\left(L^{2}\left(\mathbb{R}^{d}\right)\right)} \\
& +\sum_{l=1}^{n-1}\left\|\mathbb{1}_{\Delta_{1}}(U) S_{n, l} \mathbb{1}_{\Delta_{2}}(U)\right\|_{B(\mathcal{H}) .}
\end{aligned}
$$

The main part of the proof is to estimate $\left\|\mathbb{1}_{\Delta_{1}}(U) S_{n, l} \mathbb{1}_{\Delta_{2}}(U)\right\|_{B(\mathcal{H})}$.

Let us fix $u_{i} \in \mathbb{1}_{\Delta_{i}}(U) \mathcal{H}$ for $\Delta_{i} \Subset \mathbb{R}^{1+d}, i=1,2$. Then, recalling the definition of $a_{\underline{B}}=: a_{B_{1}, \ldots, B_{n}}$, we have

$\left(u_{1} \mid S_{n, l} u_{2}\right) \mathcal{H}$

$$
=\int\left(\psi_{1}\left(x_{1}, \ldots, x_{n-1}\right) \mid \prod_{i=1}^{l-1} B_{i}\left(y_{i}\right)\left[B_{n}^{*}\left(x_{n}\right), B_{l}\left(y_{l}\right)\right] \psi_{2}\left(y_{l+1}, \ldots, y_{n}\right)\right)_{\mathcal{H}} \prod_{i=1}^{n} \tilde{h}_{i}\left(x_{i}, y_{i}\right) d \underline{x} d \underline{y},
$$

for

$$
\begin{aligned}
& \psi_{1}\left(x_{1}, \ldots, x_{n-1}\right)=\left(a_{B_{n-1}, \ldots, B_{1}} u_{1}\right)\left(x_{n-1}, \ldots, x_{1}\right), \\
& \psi_{2}\left(y_{l+1}, \ldots, y_{n}\right)=\left(a_{B_{l+1}, \ldots, B_{n}} u_{2}\right)\left(y_{l+1}, \ldots, y_{n}\right) .
\end{aligned}
$$

Step 1: Let us first perform the integral in the variables $x_{1}, \ldots, x_{n-1}, y_{n}$. We obtain using (B.1):

$$
\begin{aligned}
& \left(u_{1} \mid S_{n, l} u_{2}\right) \mathcal{H}=\int\left(\tilde{\psi}_{1}\left(y_{1}, \ldots, y_{n-1}\right) \mid \prod_{i=1}^{l-1} B_{i}\left(y_{i}\right)\right. \\
& \left.\quad \times\left[B_{n}^{*}\left(x_{n}\right), B_{l}\left(y_{l}\right)\right] \tilde{\psi}_{2}\left(y_{l+1}, \ldots, y_{n-1}, x_{n}\right)\right)_{\mathcal{H}} \chi_{l}\left(y_{l}\right) \chi_{n}\left(x_{n}\right) d y_{1} \ldots d y_{n-1} d x_{n},
\end{aligned}
$$

for

$$
\begin{gathered}
\tilde{\psi}_{1}\left(y_{1}, \ldots, y_{n-1}\right)=\left(\left(\tilde{h}_{1}^{*} \otimes \cdots \otimes \tilde{h}_{l-1}^{*} \otimes h_{l}^{*} \otimes \tilde{h}_{l+1}^{*} \otimes \cdots \otimes \tilde{h}_{n-1}^{*}\right) \psi_{1}\right)\left(y_{1}, \ldots, y_{n-1}\right), \\
\tilde{\psi}_{2}\left(y_{l+1}, \ldots, y_{n-1}, x_{n}\right)=\left((\underbrace{\mathbb{1} \otimes \cdots \otimes \mathbb{1}}_{n-l-1} \otimes h_{n}) \psi_{2}\right)\left(y_{l+1}, \ldots, y_{n-1}, x_{n}\right) .
\end{gathered}
$$

Step 2: We now perform the integrals in $y_{1}, \ldots, y_{l-1}$.

Let us first note an easy fact: let $v_{1}=v_{1}\left(y_{1}, \ldots, y_{l-1}\right) \in \mathcal{H} \otimes L^{2}\left(\mathbb{R}^{(l-1) d}\right)$ and $v_{2} \in \mathbb{1}_{\Delta}(U) \mathcal{H}$ for $\Delta \Subset \mathbb{R}^{1+d}$. Then:

$$
\begin{aligned}
& \left|\int\left(v_{1}\left(y_{1}, \ldots, y_{l-1}\right) \mid \prod_{i=1}^{l-1} B_{i}\left(y_{i}\right) v_{2}\right) \mathcal{H} d y_{1} \ldots d y_{l-1}\right| \\
& \quad \leq\left(\int\left\|v_{1}\right\|^{2}\left(y_{1}, \ldots, y_{l-1}\right) d y_{1} \ldots d y_{l-1}\right)^{\frac{1}{2}}\left(\int\left\|\prod_{i=1}^{l-1} B_{i}\left(y_{i}\right) v_{2}\right\|_{\mathcal{H}}^{2} d y_{1} \ldots d y_{l-1}\right)^{\frac{1}{2}} \\
& \quad \leq C\left(B_{1}, \ldots, B_{l-1}\right)\left\|v_{1}\right\|_{\mathcal{H} \otimes L^{2}\left(\mathbb{R}^{(l-1) d}\right)}\left\|v_{2}\right\|_{\mathcal{H}},
\end{aligned}
$$


using successively the Cauchy-Schwarz inequality, the fact that the $B_{i}$ are energy decreasing and Lemma 3.4. Let us denote by $K\left(y_{1}, \ldots, y_{n-1}, x_{n}\right)$ the integrand in (B.3). Applying (B.4) we obtain that:

$$
\begin{aligned}
& \left|\int K\left(y_{1}, \ldots, y_{n-1}, x_{n}\right) d y_{1} \ldots d y_{l-1}\right| \\
& \quad \leq C\left(B_{1}, \ldots, B_{l-1}\right)\left(\int\left\|\tilde{\psi}_{1}\left(y_{1}, \ldots, y_{n-1}\right)\right\|_{\mathcal{H}}^{2} d y_{1} \ldots d y_{l-1}\right)^{\frac{1}{2}} \\
& \quad \times \chi_{l}\left(y_{l}\right) \chi_{n}\left(x_{n}\right)\left\|\left[B_{l}\left(y_{l}\right), B_{n}^{*}\left(x_{n}\right)\right]\right\|_{B(\mathcal{H})}\left\|\tilde{\psi}_{2}\right\|_{\mathcal{H}}\left(y_{l+1}, \ldots, y_{n-1}, x_{n}\right) .
\end{aligned}
$$

Step 3: We perform the remaining integrals in $y_{l}, \ldots, y_{n-1}, x_{n}$.

From almost locality we have $\left\|\left[B_{l}\left(y_{l}\right), B_{n}^{*}\left(x_{n}\right)\right]\right\|_{B(\mathcal{H})} \leq C_{N}\left\langle y_{l}-x_{n}\right\rangle^{-N}$. Using (3.12), we see that $\chi_{l}(y)\langle y-x\rangle^{-N} \chi_{n}(x)$ is the distributional kernel of $\chi_{l} g_{N}\left(D_{x}\right) \chi_{n}$. Using once more the Cauchy-Schwarz inequality we obtain:

$$
\begin{aligned}
& \left|\int K\left(y_{1}, \ldots, y_{n-1}, x_{n}\right) d y_{1} \ldots d y_{n-1} d x_{n}\right| \\
& \quad \leq C\left(B_{1}, \ldots, B_{l-1}\right)\left\|\tilde{\psi}_{1}\right\|_{\mathcal{H} \otimes L^{2}\left(\mathbb{R}^{(n-1) d}\right)} \times\left\|\chi_{l} g_{N}\left(D_{x}\right) \chi_{n}\right\|_{B\left(L^{2}\left(\mathbb{R}^{d}\right)\right)} \times\left\|\tilde{\psi}_{2}\right\|_{\mathcal{H} \otimes L^{2}\left(\mathbb{R}^{(n-l) d}\right)} .
\end{aligned}
$$

Now since the $B_{i}$, (resp. $\left.\psi_{1}, \psi_{2}\right)$ have compact energy-momentum transfers, (resp. spectrum), we know that:

$$
\begin{aligned}
\left\|\tilde{\psi}_{1}\right\|_{\mathcal{H} \otimes L^{2}\left(\mathbb{R}^{(n-1) d}\right)} & \leq C\left(B_{1}, \ldots, B_{n-1}\right) \prod_{i=1}^{n-1}\left\|h_{i}\right\|_{B\left(L^{2}\left(\mathbb{R}^{d}\right)\right)}\left\|u_{1}\right\|_{\mathcal{H}}, \\
\left\|\tilde{\psi}_{2}\right\| & \leq C\left(B_{l+1} \ldots, B_{n}\right)\left\|h_{n}\right\|_{B\left(L^{2}\left(\mathbb{R}^{d}\right)\right)}\left\|u_{2}\right\|_{\mathcal{H}} .
\end{aligned}
$$

Therefore we obtain that

$$
\begin{aligned}
& \left\|\mathbb{1}_{\Delta_{1}}(U) S_{n, l} \mathbb{1}_{\Delta_{2}}(U)\right\|_{B(\mathcal{H})} \\
& \quad \leq C_{N}\left(B_{1}, \ldots, B_{n}\right)\left\|\chi_{l} g_{N}\left(D_{x}\right) \chi_{n}\right\|_{B\left(L^{2}\left(\mathbb{R}^{d}\right)\right)} \prod_{i=1}^{n}\left\|h_{i}\right\|_{B\left(L^{2}\left(\mathbb{R}^{d}\right)\right)} .
\end{aligned}
$$

Step 4: Making use of (B.2) and (B.5) we obtain by induction that

$$
\begin{aligned}
& \left\|\mathbb{1}_{\Delta_{1}}(U) \tilde{R}_{n} \mathbb{1}_{\Delta_{2}}(U)\right\|_{B(\mathcal{H})} \\
& \quad \leq C_{N}(\Delta, \underline{B}) \sum_{i \neq j}\left\|\chi_{i} g_{N}\left(D_{x}\right) \chi_{j}\right\|_{B\left(L^{2}\left(\mathbb{R}^{d}\right)\right)} \prod_{i=1}^{n}\left\|h_{i}\right\|_{B\left(L^{2}\left(\mathbb{R}^{d}\right)\right)} .
\end{aligned}
$$

(We can start the induction at $n=1$, where the statement is trivial). Finally, we estimate the error terms coming from the replacement of $h_{i}$ by $\tilde{h}_{i}$. Using that the operators $a_{B_{1}, \ldots, B_{j}} \mathbb{1}_{\Delta}(U)$ are bounded, we obtain that:

$$
\begin{aligned}
& \left\|\mathbb{1}_{\Delta_{1}}(U)\left(R_{n}-\tilde{R}_{n}\right) \mathbb{1}_{\Delta_{2}}(U)\right\|_{B(\mathcal{H})} \\
& \leq C_{N}\left(B_{1}, \ldots, B_{n}\right) \times\left(\sum_{i=1}^{n}\left(\left\|h_{i}\left(1-\chi_{i}\right)\right\|_{B\left(L^{2}\left(\mathbb{R}^{d}\right)\right)}+\left\|\left(1-\chi_{i}\right) h_{i}\right\|_{B\left(L^{2}\left(\mathbb{R}^{d}\right)\right)}\right)\right. \\
& \left.\times \prod_{j \neq i}\left\|h_{j}\right\|_{B\left(L^{2}\left(\mathbb{R}^{d}\right)\right)}\right) .
\end{aligned}
$$


This completes the proof.

\section{B.2: Proof of Lemma 3.14.}

Proof. By Lemma 3.5 and (3.7), we know that

$$
\left\|N_{B_{1}}\left(h_{1}\right) \mathbb{1}_{\Delta}(U)\right\| \leq C\left\|h_{1}\right\|_{B\left(L^{2}\left(\mathbb{R}^{d}\right)\right)},\left\|B_{2}^{*}\left(g_{2}\right) \mathbb{1}_{\Delta}(U)\right\| \leq C\left\|g_{2}\right\|_{L^{2}\left(\mathbb{R}^{d}\right)} .
$$

Therefore, modulo errors controlled by the r.h.s. of (3.14), we can replace $h_{1}$ by $\tilde{h}_{1}=$ $\chi_{1} h_{1} \chi_{1}$ and $g_{2}$ by $\tilde{g}_{2}=\chi_{2} g_{2}$. Arguing as in the proof of Proposition 3.11 above, we write for $u_{i} \in \mathbb{1}_{\Delta_{i}}(U) \mathcal{H}$ :

$$
\begin{aligned}
\left(u_{1} \mid[\right. & \left.\left.N_{B_{1}}\left(\tilde{h}_{1}\right), B_{2}^{*}\left(\tilde{g}_{2}\right)\right] u_{2}\right) \mathcal{H} \\
= & \int\left(u_{1} \mid\left[B_{1}^{*}\left(x_{1}\right) B_{1}\left(y_{1}\right), B_{2}^{*}\left(x_{2}\right)\right] u_{2}\right) \tilde{H}_{1}\left(x_{1}, y_{1}\right) \tilde{g}_{2}\left(x_{2}\right) d x_{1} d y_{1} d x_{2} \\
= & \int\left(\left(\mathbb{1}_{\mathcal{H}} \otimes \tilde{h}_{1}^{*}\right) \circ a_{B_{1}} u_{1}\left(y_{1}\right) \mid\left[B_{1}\left(y_{1}\right), B_{2}^{*}\left(x_{2}\right)\right] u_{2}\right) \tilde{H}_{\mathcal{g}_{2}}\left(x_{2}\right) d y_{1} d x_{2} \\
& +\int\left(\left[B_{2}\left(x_{2}\right), B_{1}\left(x_{1}\right)\right] u_{1} \mid\left(\mathbb{1}_{\mathcal{H}} \otimes \tilde{h}_{1}\right) \circ a_{B_{1}} u_{2}\left(x_{1}\right)\right)_{\mathcal{H}} \tilde{g}_{2}\left(x_{2}\right) d x_{1} d x_{2} \\
= & I_{1}+I_{2} .
\end{aligned}
$$

Using Cauchy-Schwarz and almost locality we obtain:

$\left|I_{1}\right| \leq C_{N} \int\left\|\left(\mathbb{1}_{\mathcal{H}} \otimes h_{1}^{*} \chi_{1}\right) \circ a_{B_{1}} u_{1}\right\|_{\mathcal{H}}\left(y_{1}\right) \chi_{1}\left(y_{1}\right)\left\langle y_{1}-x_{2}\right\rangle^{-N} \chi_{2}\left(x_{2}\right)\left|g_{2}\right|\left(x_{2}\right)\left\|u_{2}\right\|_{\mathcal{H}} d y_{1} d x_{2}$,
$\left|I_{2}\right| \leq C_{N} \int\left\|\left(\mathbb{1}_{\mathcal{H}} \otimes \chi_{1} h_{1}\right) \circ a_{B_{1}} u_{2}\right\|_{\mathcal{H}}\left(x_{1}\right) \chi_{1}\left(x_{1}\right)\left\langle x_{1}-x_{2}\right\rangle^{-N} \chi_{2}\left(x_{2}\right)\left|g_{2}\right|\left(x_{2}\right)\left\|u_{1}\right\|_{\mathcal{H}} d y_{1} d x_{2}$. Applying once more Lemma 3.5 we obtain

$\left|I_{1}\right|+\left|I_{2}\right| \leq C_{N}\left(\Delta_{1}, \Delta_{2}\right)\left\|h_{1}\right\|_{B\left(L^{2}\left(\mathbb{R}^{d}\right)\right)}\left\|u_{1}\right\|_{\mathcal{H}}\left\|u_{2}\right\|_{\mathcal{H}}\left\|\chi_{1} g_{N}\left(D_{x}\right) \chi_{2}\right\|_{B\left(L^{2}\left(\mathbb{R}^{d}\right)\right)}\left\|g_{2}\right\|_{L^{2}\left(\mathbb{R}^{d}\right)}$, which completes the proof of the lemma.

\section{B.3: Proof of Theorem 6.5.}

Proof. (1). Let $B_{i}, g_{i}$ be as specified in the theorem. First we show that for $i \neq j$

$$
\left[B_{i, t}^{(*)}\left(g_{i, t}\right), B_{j, t}^{(*)}\left(g_{j, t}\right)\right] \in O\left(t^{-\infty}\right) .
$$

By Proposition 6.2 we can find functions $\chi_{i}, \chi_{j} \in C_{0}^{\infty}\left(\mathbb{R}^{d}\right)$ with disjoint supports such that

$$
g_{i, t}=\chi_{i}\left(\frac{x}{t}\right) g_{i, t}+O\left(t^{-\infty}\right) \text { in } L^{2}\left(\mathbb{R}^{d}\right),
$$

and similarly for $g_{j, t}$. We set $\chi_{i, t}(x):=\chi_{i}\left(\frac{x}{t}\right), \chi_{j, t}(x):=\chi_{j}\left(\frac{x}{t}\right)$ and note that by Lemma 6.4 (2) for any compact set $\Delta$

$$
\left[B_{i, t}^{(*)}\left(g_{i, t}\right), B_{j, t}^{(*)}\left(g_{j, t}\right)\right] \mathbb{1}_{\Delta}(U)=\left[B_{i, t}^{(*)}\left(\chi_{i, t} g_{i, t}\right), B_{j, t}^{(*)}\left(\chi_{j, t} g_{j, t}\right)\right] \mathbb{1}_{\Delta}(U)+O\left(t^{-\infty}\right) .
$$


Using almost locality of $B_{i}^{(*)}, B_{j}^{(*)}$ we obtain from (3.4) that the commutator in the r.h.s. is of order $O\left(t^{-\infty}\right)$, which proves (B.7). Now we can write

$$
\partial_{t}\left(B_{1, t}^{*}\left(g_{1, t}\right) \ldots B_{n, t}^{*}\left(g_{n, t}\right)\right) \Omega=\sum_{i=1}^{n} B_{1, t}^{*}\left(g_{1, t}\right) \ldots \partial_{t}\left(B_{i, t}^{*}\left(g_{i, t}\right)\right) \ldots B_{n, t}^{*}\left(g_{n, t}\right) \Omega .
$$

Due to Lemma $6.4(1), \partial_{t}\left(B_{i, t}^{*}\left(g_{i, t}\right)\right)$ annihilates the vacuum. Thus we commute this expression to the right until it acts on the vacuum and show that the resulting terms with the commutators are $O\left(t^{-\infty}\right)$. This follows from (B.7) and from Lemma 6.4 (2),(3). Using the Cook argument we conclude the proof of (1).

Before we proceed to the proof of (2), we need some preparation: Let $B \in \mathcal{L}_{0}$ satisfy (2.11), and $\Delta=-\operatorname{supp}(\widehat{B}) \cap \mathcal{S} p U \subset H_{m}$. We fix $O \subset \mathbb{R}^{1+d}$, which is an arbitrarily small neighborhood of $\Delta$, and a function $h \in \mathcal{S}\left(\mathbb{R}^{1+d}\right)$ with supp $\widehat{h} \subset O$ and $\widehat{h}=(2 \pi)^{-(1+d) / 2}$ on $\Delta$. Then $C^{*}:=\int B^{*}(t, x) h(t, x) d t d x$ is an element of $\mathcal{L}_{0}$ and

$$
\widehat{C^{*}}(E, p)=(2 \pi)^{(1+d) / 2} \widehat{h}(E, p) \widehat{B^{*}}(E, p), C^{*} \Omega=(2 \pi)^{(1+d) / 2} \widehat{h}(H, P) B^{*} \Omega .
$$

Consequently $-\operatorname{supp}(\widehat{C}) \subset O$, and

$$
\begin{aligned}
B_{t}^{*}\left(g_{t}\right) \Omega & =(2 \pi)^{d / 2} \widehat{f}(P) B^{*} \Omega=(2 \pi)^{d / 2} \widehat{f}(P) \mathbb{1}_{\Delta}(U) B^{*} \Omega \\
& =(2 \pi)^{d / 2} \widehat{f}(P)(2 \pi)^{(1+d) / 2} \widehat{h}(H, P) B^{*} \Omega=(2 \pi)^{d / 2} \widehat{f}(P) C^{*} \Omega=C_{t}^{*}\left(g_{t}\right) \Omega .
\end{aligned}
$$

We define observables $C_{i}$ corresponding to $B_{i}$ and obtain

$$
\Psi^{+}=\lim _{t \rightarrow \infty} B_{1, t}^{*}\left(g_{1, t}\right) \ldots B_{n, t}^{*}\left(g_{n, t}\right) \Omega=\lim _{t \rightarrow \infty} C_{1, t}^{*}\left(g_{1, t}\right) \ldots C_{n, t}^{*}\left(g_{n, t}\right) \Omega,
$$

where we used (B.7) and Lemma 6.4 (2). Therefore we can assume that the energymomentum transfers of $B_{i}^{*}$ entering in the construction of scattering states are localized in arbitrarily small neighborhoods of subsets of $H_{m}$.

(2). Let $\tilde{\Psi}_{t}=\tilde{B}_{1, t}^{*}\left(\tilde{g}_{1, t}\right) \ldots \tilde{B}_{n, t}^{*}\left(\tilde{g}_{n, t}\right) \Omega$ be the approximating sequence of the scattering state $\tilde{\Psi}^{+}$. In order to analyse the scalar product $\left(\tilde{\Psi}_{t} \mid \Psi_{t}\right)$ we first show that

$$
\left[\left[\tilde{B}_{j, t}\left(\tilde{g}_{j, t}\right), B_{k, t}^{*}\left(g_{k, t}\right)\right], B_{l, t}^{*}\left(g_{l, t}\right)\right] \in O\left(t^{-\infty}\right), \quad k \neq l .
$$

To verify (B.11) we write $\tilde{g}_{j}=\tilde{g}_{j, k}+\tilde{g}_{j, l}$, where $\tilde{g}_{j, k}, \tilde{g}_{j, l}$ are positive energy KG solutions such that $\tilde{g}_{j, i}$ and $g_{i}$ have disjoint velocity supports for $i=k, l$. Then (B.11) follows from (B.7) and the Jacobi identity.

Next we note that

$$
\tilde{B}_{i, t}\left(\tilde{g}_{i, t}\right) B_{j, t}^{*}\left(g_{j, t}\right) \Omega=\Omega\left(\Omega \mid \tilde{B}_{i, t}\left(\tilde{g}_{i, t}\right) B_{j, t}^{*}\left(g_{j, t}\right) \Omega\right), \quad i, j=1, \ldots, n .
$$

(B.12) follows from the fact that $\tilde{B}_{i, t}\left(\tilde{g}_{i, t}\right) B_{j, t}^{*}\left(g_{j, t}\right) \Omega$ belongs to the range of $\mathbb{1}_{-K_{j}+\tilde{K}_{i}}(U)$, where $K_{j}$ and $\tilde{K}_{i}$ are the energy-momentum transfers of $B_{j}$ and $\tilde{B}_{i}$, respectively. Due to (B.10) $-K_{j},-\tilde{K}_{i}$ can be chosen in arbitrarily small neighbourhoods of $H_{m}$. Since a non-zero vector which is a difference of two vectors from $H_{m}$ is spacelike, (B.12) follows. 
To prove (6.2), we set for simplicity of notation $B_{i}(t):=\left(B_{i, t}^{*}\left(g_{i, t}\right)\right)^{*}, \tilde{B}_{j}(t):=$ $\left(\tilde{B}_{j, t}^{*}\left(\tilde{g}_{j, t}\right)\right)^{*}$. We assume that (6.2) holds for $n-1$ and compute

$$
\begin{aligned}
\left(\tilde{\Psi}_{t} \mid \Psi_{t}\right)= & \left(\Omega \mid \tilde{B}_{n}(t) \ldots \tilde{B}_{1}(t) B_{1}^{*}(t) \ldots B_{n}^{*}(t) \Omega\right) \\
= & \sum_{k=1}^{n}\left(\Omega \mid \tilde{B}_{n}(t) \ldots \tilde{B}_{2}(t) B_{1}^{*}(t) \ldots\left[\tilde{B}_{1}(t), B_{k}^{*}(t)\right] \ldots B_{n}^{*}(t) \Omega\right) \\
= & \sum_{k=1}^{n} \sum_{l=k+1}^{n}\left(\Omega \mid \tilde{B}_{n}(t) \ldots \tilde{B}_{2}(t) B_{1}^{*}(t) \ldots \check{k} \ldots\left[\left[\tilde{B}_{1}(t), B_{k}^{*}(t)\right], B_{l}^{*}(t)\right] \ldots B_{n}^{*}(t) \Omega\right) \\
& +\sum_{k=1}^{n}\left(\Omega \mid \tilde{B}_{n}(t) \ldots \tilde{B}_{2}(t) B_{1}^{*}(t) \ldots \check{k} \ldots B_{n}^{*}(t) \Omega\right)\left(\Omega \mid \tilde{B}_{1}(t) B_{k}^{*}(t) \Omega\right), \quad(\mathrm{B} .13)
\end{aligned}
$$

where in the last term on the r.h.s. we applied (B.12). Now we note that the last term factorizes in the limit $t \rightarrow \infty$ by the induction hypothesis and the terms involving double commutators vanish by (B.11).

It is an immediate consequence of (6.2) that the scattering states $\Psi^{+}$depend only on the single-particle states $\Psi_{i}$ (and not on a particular choice of $B_{i}$ and $g_{i}$ ). Relation (6.3) follows from Lemma 6.4 (1).

\section{References}

[AH67] Araki, H., Haag, R.: Collision cross sections in terms of local observables. Commun. Math. Phys. 4, 77-91 (1967)

[Ar99] Araki, H.: Mathematical Theory of Quantum Fields. Oxford Science Publications, Oxford (1999)

[Ar82] Arveson, W.: The harmonic analysis of automorphism groups. In: Operator Algebras and Applications, Part I (Kingston, Ont., 1980), Proc. Sympos. Pure Math., vol. 38. Amer. Math. Soc., Providence, pp. 199-269 (1982)

[BFG78] Béllisard, J., Fröhlich, J., Gidas, B.: Soliton mass and surface tension in the $\left(\lambda \phi^{4}\right)_{2}$ quantum field model. Commun. Math. Phys. 60, 37-72 (1978)

[Bu90] Buchholz, D.: Harmonic analysis of local operators. Commun. Math. Phys. 129, 631-641 (1990)

[BF82] Buchholz, D., Fredenhagen, K.: Locality and the structure of particle states. Commun. Math. Phys. 84, 1-54 (1982)

[Bu87] Buchholz, D.: Particles, infraparticles and the problem of asymptotic completeness. In: VIIIth International Congress on Mathematical Physics (Marseille 1986). Word Scientific, Singapore (1987)

[Bu94] Buchholz, D.: On the manifestations of particles. In: Mathematical Physics Towards the 21st Century. In: Sen, R.N., Gersten, A. (eds.) Proceedings Beer-Sheva 1993. Ben-Gurion University of the Negev Press (1994)

[BS05] Buchholz, D., Summers, S.J: Scattering in relativistic quantum field theory: fundamental concepts and tools. In: Françoise, J.-P., Naber, G., Tsun, T.S. (eds.) Encyclopedia of Mathematical Physics, vol. 5, pp. 1. Elsevier, Amsterdam (2006)

[Bur77] Burnap, C.: Isolated one particle states in boson quantum field theory models. Ann. Phys. 104, 184196 (1977)

[CD82] Combescure, M., Dunlop, F.: Three-body asymptotic completeness for $P(\phi)_{2}$ models. Commun. Math. Phys. 85, 381-418 (1982)

[DG97] Dereziński, J., Gérard, C.: Scattering Theory of Classical and Quantum N-Particle Systems. Springer, New York (1997)

[DHR71] Doplicher, S., Haag, R., Roberts, J.E.: Local observables and particle statistics I. Commun. Math. Phys. 23, 199-230 (1971)

[DHR74] Doplicher, S., Haag, R., Roberts, J.E.: Local observables and particle statistics II. Commun. Math. Phys. 35, 49-85 (1974)

[Dy05] Dybalski, W.: Haag-Ruelle scattering theory in presence of massless particles. Lett. Math. Phys. 72, 27-38 (2005) 
[DG12] Dybalski, W., Gérard, C.: Towards asymptotic completeness of two-particle scattering in local relativistic QFT. Commun. Math. Phys. 326(i), 81-109 (2014)

[DT11a] Dybalski, W., Tanimoto, Y.: Asymptotic completeness for infraparticles in two-dimensional conformal field theory. Lett. Math. Phys. 103(ii), 1223-1241 (2013)

[De93] Dereziński, J.: Asymptotic completeness of long-range $n$-body quantum systems. Ann. Math. 138, 427-476 (1993)

[FRS89] Fredenhagen, K., Rehren, K.H., Schroer, B.: Superselection sectors with braid group statistics and exchange algebras I. General theory. Commun. Math. Phys. 125, 201-226 (1989)

[Fr76] Fröhlich, J.: New super-selection sectors ("soliton-states") in two-dimensional bose quantum field models. Commun. Math. Phys. 47, 269-310 (1976)

[Ge91] Gérard, C.: Mourre estimate for regular dispersive systems. Ann. Inst. H. Poincaré 54, 5988 (1991)

[GJS73] Glimm, J., Jaffe, A., Spencer, T.: The particle structure of the weakly coupled $P(\phi)_{2}$ model and other applications of high temperature expansions: part I. Physics of quantum field models. Part II. The cluster expansion. In: Velo, G., Wightman, A.S. (eds.) Constructive Quantum Field Theory. (Erice, 1973). Springer, Berlin (1973)

[GJS74] Glimm, J., Jaffe, A., Spencer, T.: The Wightman axioms and particle structure in the $P(\phi)_{2}$ quantum field model. Ann. Math. 100, 585-632 (1974)

[Gr90] Graf, G.M.: Asymptotic completeness for n-body short-range quantum systems: a new proof. Commun. Math. Phys. 132, 73-101 (1990)

[Gre61] Greenberg, O.W.: Generalized free fields and models of local field theory. Ann. Phys. 16, 158176 (1961)

[Ha58] Haag, R.: Quantum field theories with composite particles and asymptotic conditions. Phys. Rev. 112, 669-673 (1958)

[Ha] Haag, R.: Local Quantum Physics. Springer, New York (1992)

[HS65] Haag, R., Swieca, J.A.: When does a quantum field theory describe particles? Commun. Math. Phys. 1, 308-320 (1965)

[He65] Hepp, K.: On the connection between the LSZ and Wightman quantum field theory. Commun. Math. Phys. 1, 95-111 (1965)

[He71] Herbst, I.: One-particle operators and local internal symmetries. J. Math. Phys. 12, 2480-2490 (1971)

[Ho85] Hörmander, L.: The Analysis of Linear Partial Differential Operators III. Springer, Berlin (1985)

[Le08] Lechner, G.: Construction of quantum field theories with factorizing S-matrices. Commun. Math. Phys. 277, 821-860 (2008)

[Mu98] Müger, M.: Superselection structure of massive quantum field theories in $1+1$ dimensions. Rev. Math. Phys. 10, 1147-1170 (1998)

[Ru62] Ruelle, D.: On the asymptotic condition in quantum field theory. Helv. Phys. Acta 35, 147163 (1962)

[RS3] Reed, M., Simon, B.: Methods of Modern Mathematical Physics. Part III: Scattering Theory. Academic Press, New York (1979)

[SiSo87] Sigal, I.M., Soffer, A.: The n-particle scattering problem: asymptotic completeness for short-range systems. Ann. Math. 126, 35-108 (1987)

[SZ76] Spencer, T., Zirilli, F.: Scattering states and bound states in $\lambda P(\phi)_{2}$. Commun. Math. Phys. 49, 116 (1976)

[SW] Streater, R.F., Wightman, A.S.: PCT, Spin and Statistics and All That. Princeton University Press, Princeton (2000)

[Ta13] Tanimoto, Y.: Construction of two-dimensional quantum field models through Longo-Witten endomorphisms. arXiv:1301.6090 [math-ph] (2013, preprint)

[Zi97] Zieliński, L.: Scattering for a dispersive charge-transfer model. Ann. Inst. Henri Poincaré 67, 339_ 386 (1997)

Communicated by Y. Kawahigashi 